\title{
Deterministic Drift Counteraction Optimal Control for Attitude Control of Spacecraft with Time-Varying Mass
}

\author{
Robert A. E. Zidek* and Ilya V. Kolmanovsky ${ }^{\dagger}$ \\ University of Michigan, Ann Arbor, MI 48109, USA
}

\begin{abstract}
A solution method for a special class of deterministic drift counteraction optimal control (DDCOC) problems is presented. The objective is to find a control law that maximizes the time until a deterministic nonlinear discrete-time system violates prescribed constraints. One of the system's states is monotonically decreasing in time, decreasing by a constant rate each time a control action is applied. This, for example, may model the propellant mass of spacecraft propulsion systems that operate with a constant mass flow. The method is applied to attitude control of an axisymmetric spacecraft during a translational thrusting maneuver with a fixed thrust vector misalignment. The spacecraft's mass and inertia properties are time-varying due to the mass flow required for the orbital maneuver. Given fuel constraints for the attitude control system, the objective is to counteract the parasitic moment resulting from the thrust vector misalignment and to maximize the time during which the orientation of the spacecraft's symmetry axis stays within a prescribed cone. Simulation results are presented including a robustness analysis of the DDCOC law with respect to uncertainties in the thrust vector misalignment.
\end{abstract}

\section{Nomenclature}

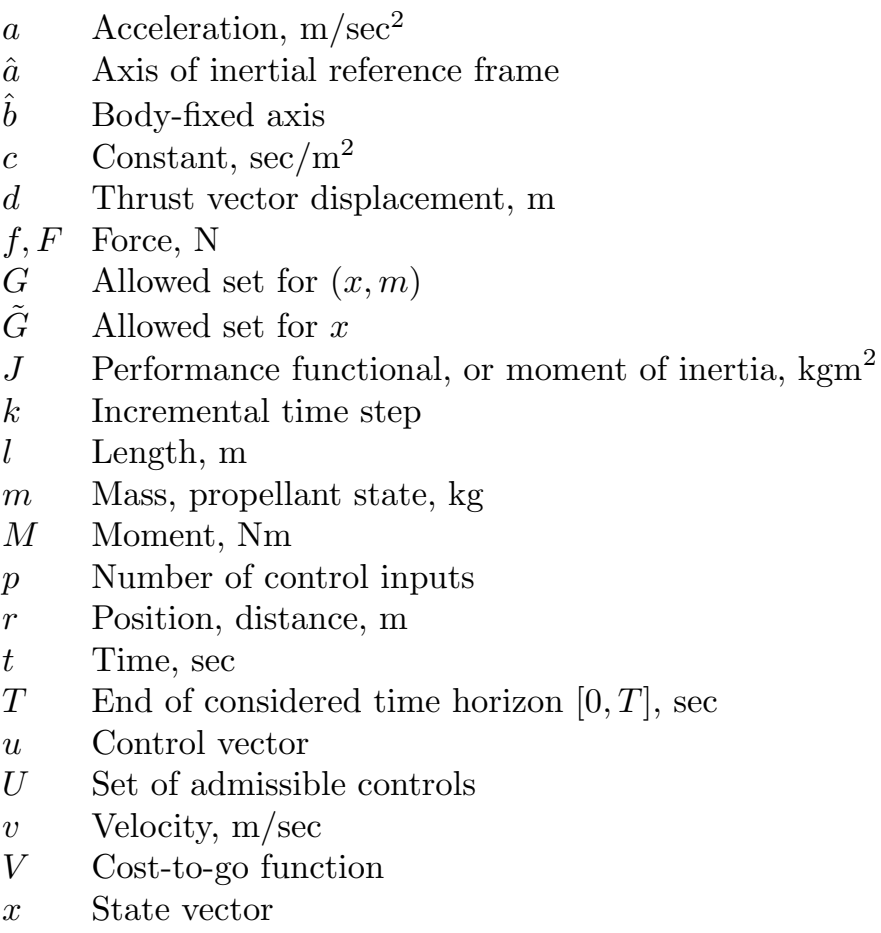

* PhD Candidate, Department of Aerospace Engineering, AIAA member

${ }^{\dagger}$ Professor, Department of Aerospace Engineering, AIAA member 
$\alpha \quad$ Maximum control input

$\beta \quad$ Thrust vector misdirection, deg

$\Delta t \quad$ Sampling time, sec

$\theta \quad$ Attitude parameter

$\rho \quad$ Density, $\mathrm{kg} / \mathrm{m}^{3}$

$\tau \quad$ First instant at which $(x, m) \notin G$

$\omega$ Angular velocity, $1 / \mathrm{sec}$

Subscript

A Inertial reference frame

B Body-fixed frame

c Center of mass

$\mathcal{B}$ General rigid body or spacecraft

e Main engine

f Fuel

$k \quad$ Time instant

ox Oxidizer

p Payload

$\mathrm{R} \quad$ Moment of inertia component about symmetry axis

$\mathrm{t}$ Thrust from main engine

T Moment of inertia component about principal axes 1 and 2

$z \quad$ Body-fixed point

\section{Introduction}

The framework of drift counteraction optimal control (DCOC) may be used to find a control law that maximizes the time or total yield until a system violates prescribed constraints. The systems typically considered for DCOC are driven by a large disturbance with limited resources available for counteracting the disturbance. DCOC has been previously considered for stochastic systems ${ }^{1-3}$ leading to stochastic DCOC (SDCOC) and recently for deterministic systems (DDCOC) as well. ${ }^{4}$ Both SDCOC and DDCOC are based on dynamic programming (DP) and the solution can be computed (off-line) by the usual DP numerical algorithms such as value iteration. ${ }^{2,5}$ Problems similar to DCOC are discussed, for example, by Bertsekas ${ }^{6}$ or by Blanchini and Miani ${ }^{7}$ who use set bounding methods. Their approaches are based on DP as well. In contrast to previous work, DCOC assumes that the disturbance acting on the system is sufficiently large such that the system will violate prescribed constraints at an unknown finite time.

The computational effort to solve the DCOC problem using conventional DP algorithms increases exponentially with the dimension of the system. This is also known as the curse of dimensionality which may be dealt with using approximate dynamic programming (ADP) techniques. ${ }^{8,9}$ The ADP is based on a priori assumptions about the optimal control or value function.

In this paper a new solution method is presented for a special class of problems which allows to generate DCOC-based control laws faster than common DP algorithms. Moreover, the DCOC framework is applied to a time-varying system of higher order than in previous work. The systems that are considered have a state $m$ which is monotonically nonincreasing in time, where $m$ decreases by a constant rate each time a control action is applied.

The example application in this paper is an attitude control problem for an axisymmetric spacecraft. The system is time-varying and has five continuous states. Four states are used to describe the rotational dynamics of the spacecraft and its orientation. The fifth state $m$ describes the available propellant mass for the attitude control system.

The spacecraft is performing a longitudinal thrusting maneuver to change its orbit. The accuracy of such orbital maneuvers depends on the orientation of the spacecraft during the thrusting period. Due to imperfections and varying mass properties the thrust vector may be misaligned relative to the spacecraft's center of mass. This creates a disturbance moment trying to rotate the spacecraft. There are principally two approaches for active closed-loop control of the spacecraft's attitude during thrusting maneuvers: fixed thrust and thrust vector control. ${ }^{10}$ Thrust vector control is achieved using gimbaled engines or thrust deflection devices. Fixed thrust systems are less complex than thrust vector control systems. However, they require 
sufficient control authority to counteract the parasitic moment resulting from the thrust vector misalignment. For the example in this paper we assume a fixed thrust system with attitude control thrusters. The DDCOC law is used to maximizes the time that the spacecraft's symmetry axis stays inside a prescribed cone given limited amount of propellant for attitude control. The DDCOC law takes into account the time-varying mass and inertia properties of the spacecraft resulting from the propellant consumption required for the orbital maneuver.

The structure of the paper is as follows. The general DDCOC problem is described in Section 2. Section 3 presents the novel approach to solve the DDCOC problem. The spacecraft attitude control example is discussed in Section 4. This includes a robustness analysis of the DDCOC law with respect to uncertainties in the thrust vector misalignment. The rotational dynamics of a spacecraft with time-varying mass and inertia properties are derived in the Appendix. Section 5 provides a brief conclusion and a discussion of future work.

\section{DDCOC Problem Formulation}

We consider a class of time-varying nonlinear discrete-time systems of the form

$$
\begin{aligned}
& x_{k+1}=f\left(x_{k}, u_{k}, t_{k}\right), \\
& m_{k+1}=m_{k}-c \sum_{i=1}^{p}\left|u_{k, i}\right|=m_{k}-c\left\|u_{k}\right\|_{1},
\end{aligned}
$$

where $\|\cdot\|_{1}$ denotes the 1 -norm and $k \in \mathbb{Z}_{\geq 0}$. The time is denoted by $t_{k} \geq 0$ which satisfies $t_{k+1}=t_{k}+\Delta t$, where $\Delta t$ is the sampling time of the discrete-time system. The states of the system are $x_{k} \in \mathbb{R}^{n-1}$ and $m_{k} \in \mathbb{R}$. The parameter $c>0$ is a real constant. The control input is $u_{k}=\left[u_{k, 1}, u_{k, 2}, \ldots, u_{k, p}\right]^{\mathrm{T}} \in U \subset \mathbb{R}^{p}$, where

$$
u_{k, i} \in\{-\alpha, 0, \alpha\}, \quad i=1,2, \ldots, p, \quad \alpha \in \mathbb{R} .
$$

The function $f$ in Eq. (1) can be any nonlinear function mapping from $\mathbb{R}^{n-1} \times U \times \mathbb{R}_{\geq 0}$ into $\mathbb{R}^{n-1}$. The objective is to find a control law $u: G \times \mathbb{R}_{\geq 0} \rightarrow U$ such that the following performance criterion is maximized

$$
J=\sum_{k=0}^{\tau^{x_{0}, m_{0}, t_{0}, u}(G)-1} 1=\tau^{x_{0}, m_{0}, t_{0}, u}(G),
$$

where $\tau^{x_{0}, m_{0}, t_{0}, u}(G)$ is the first instant at which the system exits a specified compact set $G$ given the initial condition $x_{0}, m_{0}, t_{0}$, and the control law $u$. The set $G \subset \mathbb{R}^{n}$ is defined as

$$
G=\left\{(x, m): x \in \tilde{G}, m \geq m_{\min }\right\},
$$

with $\tilde{G} \subset \mathbb{R}^{n-1}$ and $m_{\min } \in \mathbb{R}$. The optimal control policy $u^{*}$ satisfies the sufficient conditions stated in the following theorem.

Theorem 1. Suppose there exists a non-negative function $V: \mathbb{R}^{n-1} \times \mathbb{R} \times \mathbb{R}_{\geq 0} \rightarrow \mathbb{R}_{\geq 0}$ that is bounded from above satisfying, for all $x \in \mathbb{R}^{n-1}, m \in \mathbb{R}$, and $t \geq 0$,

$$
\begin{aligned}
& V(x, m, t)-V\left(f\left(x, u^{*}(x, m, t), t\right), m-c\left\|u^{*}(x, m, t)\right\|_{1}, t+\Delta t\right)=1, \text { if }(x, m) \in G, \\
& V(x, m, t)-V\left(f(x, u(x, m, t), t), m-c\|u(x, m, t)\|_{1}, t+\Delta t\right) \geq 1, \text { if }(x, m) \in G, u \neq u^{*}, \\
& V(x, m, t)=0, \text { if }(x, m) \notin G .
\end{aligned}
$$

Then the control policy $u^{*}$ maximizes Eq. (3). Furthermore, $V\left(x_{0}, m_{0}, t_{0}\right)=\tau^{x_{0}, m_{0}, t_{0}, u^{*}}(G)$ and $V\left(x_{k}, m_{k}, t_{k}\right)=$ $\tau^{x_{0}, m_{0}, t_{0}, u^{*}}(G)-k, k \in \mathbb{Z}_{\geq 0}$.

Proof. The proof for time-varying systems is similar to the proof for time-invariant systems by Zidek and Kolmanovsky. ${ }^{4}$ Using any sub-optimal control policy $u$, it follows from (5) that, for all $t \geq 0$,

$$
V(x, m, t)-V\left(x_{\tau}, m_{\tau}, t_{\tau}\right) \geq \tau,
$$


where $\tau=\tau^{x, m, t, u}(G)$. Since $V\left(x_{\tau}, m_{\tau}, t_{\tau}\right)=0$, it follows from Eq. (6) that

$$
V(x, m, t) \geq \tau .
$$

Defining $\tau^{*}=\tau^{x, m, t, u^{*}}(G)$ and using the optimal control law $u^{*}$ as well as the fact that $V\left(x_{\tau^{*}}, m_{\tau^{*}}, t_{\tau^{*}}\right)=0$, it can be shown that

$$
V(x, m, t)=\tau^{*}
$$

Comparing Eq. (7) and (8), it follows that $\tau^{*} \geq \tau$ or $\tau^{x, m, t, u^{*}}(G) \geq \tau^{x, m, t, u}(G)$.

Conditions under which we can expect the existence of a function $V$ satisfying the requirements stated in Theorem 1 were presented by Zidek and Kolmanovsky. ${ }^{4}$ For example, it is sufficient that for all $(x, m) \in G$ and $t \geq 0, \tau^{x, m, t, u}(G)$ is finite for any control policy $u$.

\section{Solving the DDCOC Problem}

According to (5), the optimal control law is given by

$$
u^{*}(x, m, t) \in \underset{u \in U}{\operatorname{argmax}} V\left(f(x, u, t), m-c\|u\|_{1}, t+\Delta t\right) .
$$

Therefore, the solution of the DDCOC problem is the function $V$ that satisfies (5). This function is also referred to as the cost-to-go function or value function. An exact solution for $V$ may be obtained using the value iteration algorithm ${ }^{2}$ or a variant of it. ${ }^{4}$ However, these algorithms become computationally intractable for higher-dimensional systems.

Remark 1. If the maximizer in Eq. (9) is not unique we select a maximizer that requires the least control effort in the sense that $\|u\|_{1}$ is minimal.

\section{A. Computing Optimal Control}

A solution method different from value iterations is developed for systems given by Eq. (1) based on the following idea. According to Eq. (1) and (2) the state $m$ decreases by multiples of a constant value $c \alpha$ each time a non-zero control action is applied. Therefore, we discretize $m$ by $m_{\min }+q c \alpha, q \in \mathbb{Z}_{\geq 0}$, yielding the following grid

$$
m \in \mathcal{M}_{\mathrm{dis}}=\left\{m_{\min }, m_{\min }+c \alpha, m_{\min }+2 c \alpha, m_{\min }+3 c \alpha, \ldots\right\} .
$$

Then, assuming that $V\left(x_{0}, m_{\min }, t_{0}\right)$ is known, $V\left(x_{0}, m_{\min }+c \alpha, t_{0}\right)$ is obtained by finding the state and time on the zero-control trajectory $(u=0)$ at which it is optimal to apply a control action. This is done by traversing the zero-control trajectory emanating from $x_{0}$ at time $t_{0}$ until the constraints are violated. At each point of this zero-control trajectory we compute

$$
V_{k-1}=\max _{u \in U}\left\{V\left(f\left(x_{k-1}, u, t_{k-1}\right), m_{\min }, t_{k-1}+\Delta t\right)\right\}+k,
$$

where $k \in \mathbb{Z}_{+}$increases incrementally at each time instant. Then $V\left(x_{0}, m_{\min }+c \alpha, t_{0}\right)=\max _{k \in \mathbb{Z}_{>0}} V_{k}$. After $V\left(x_{0}, m_{\min }+c \alpha, t_{0}\right)$ is computed, $V\left(x_{0}, m_{\min }+2 c \alpha, t_{0}\right)$ can be obtained following the same idea. The same applies to $V\left(x_{0}, m_{\min }+3 c \alpha, t_{0}\right)$ and so on. It can be shown that the resulting $V$ satisfies the sufficient conditions for optimality stated in (5).

For numerical implementation of this algorithm, the spaces of the state vector $x$ and of the time $t$ have to be discretized in addition to discretizing the state $m$, see Eq. (10). Moreover, since $V(x, m, t)=0$ for $x \notin \tilde{G}$, we only consider $x \in \tilde{G}$. The discretized state space for $x$ is denoted by $\tilde{G}_{\text {dis }} \subset \tilde{G}$ and the discretized time is denoted by $\mathcal{T}_{\text {dis }} \subset[0, T]$, where $T$ is the end of the considered time horizon.

The algorithm is based on the knowledge of $V\left(x, m_{\min }, t\right)$. Computing $V\left(x, m_{\min }, t\right)$ is straightforward by noting that a non-zero control action would violate the constraints given by Eq. (4) since $m=m_{\min }$. Thus, we traverse the zero-control trajectory starting from $x \in \tilde{G}$, where $V\left(x, m_{\min }, t\right)$ is the number of steps $k$ until $x \notin \tilde{G}$. This procedure is summarized in Algorithm 1.

The new approach computes the DDCOC solution significantly faster than conventional DP algorithms if $\tau^{x, m_{\min }, t, 0}(G)$ or $V\left(x, m_{\min }, t\right)$, respectively, are sufficiently small for all $x \in \tilde{G}_{\text {dis }}$ and $t \in \mathcal{T}_{\text {dis }}$. This is the case when the disturbance acting on the system is large or the constraints given by $\tilde{G}$ are tight. 


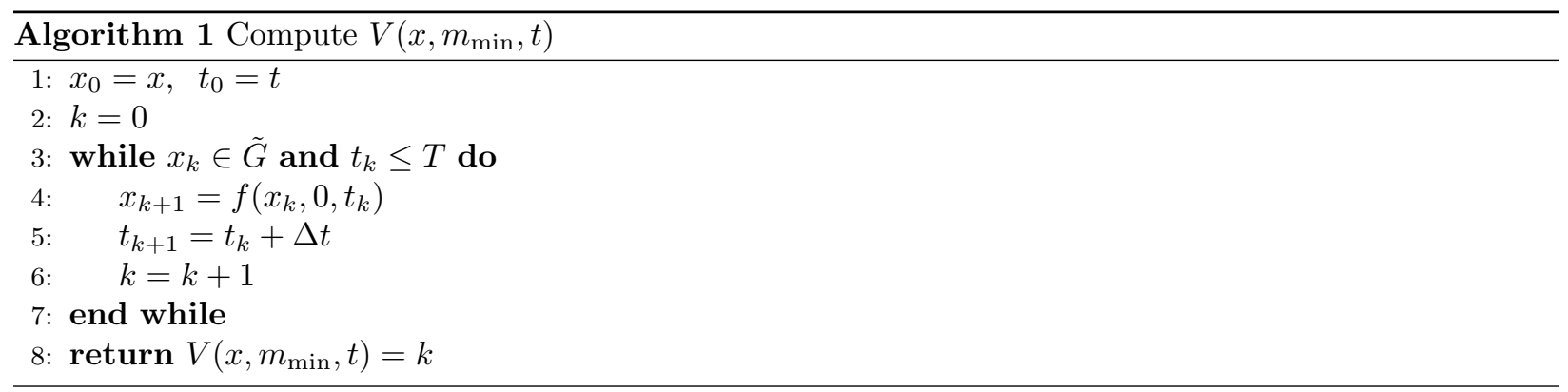

As outlined above, knowing $V\left(x, m_{\min }, t\right)$ for all $x \in \tilde{G}_{\text {dis }}$ and $t \in \mathcal{T}_{\text {dis }}$ allows to compute $V\left(x, m_{\min }+\right.$ $q c \alpha, t)$ for all $q \in \mathbb{Z}_{+}$. However, care needs to be taken because multi-input systems with $u \in U \subset \mathbb{R}^{p}$ are considered. For example, if $q<p$ it is not possible to apply a control with $u_{i} \neq 0$ for all $i=1,2, \ldots, p$ because this would violate the constraint $m \geq m_{\min }$. Therefore, the following set is defined

$$
U_{q}=\left\{u \in U: \sum_{i=1}^{p}\left|u_{i}\right| \leq q \alpha\right\}, \quad q \in \mathbb{Z}_{+} .
$$

Thus, the maximizer in Eq. (9) is selected from the set $U_{q}$ instead of $U$. With the optimality conditions in (5) and the ideas outlined before, we can now state Algorithm 2 to compute $V\left(x, m_{\min }+q c \alpha, t\right)$ for all $q \in \mathbb{Z}_{+}$.

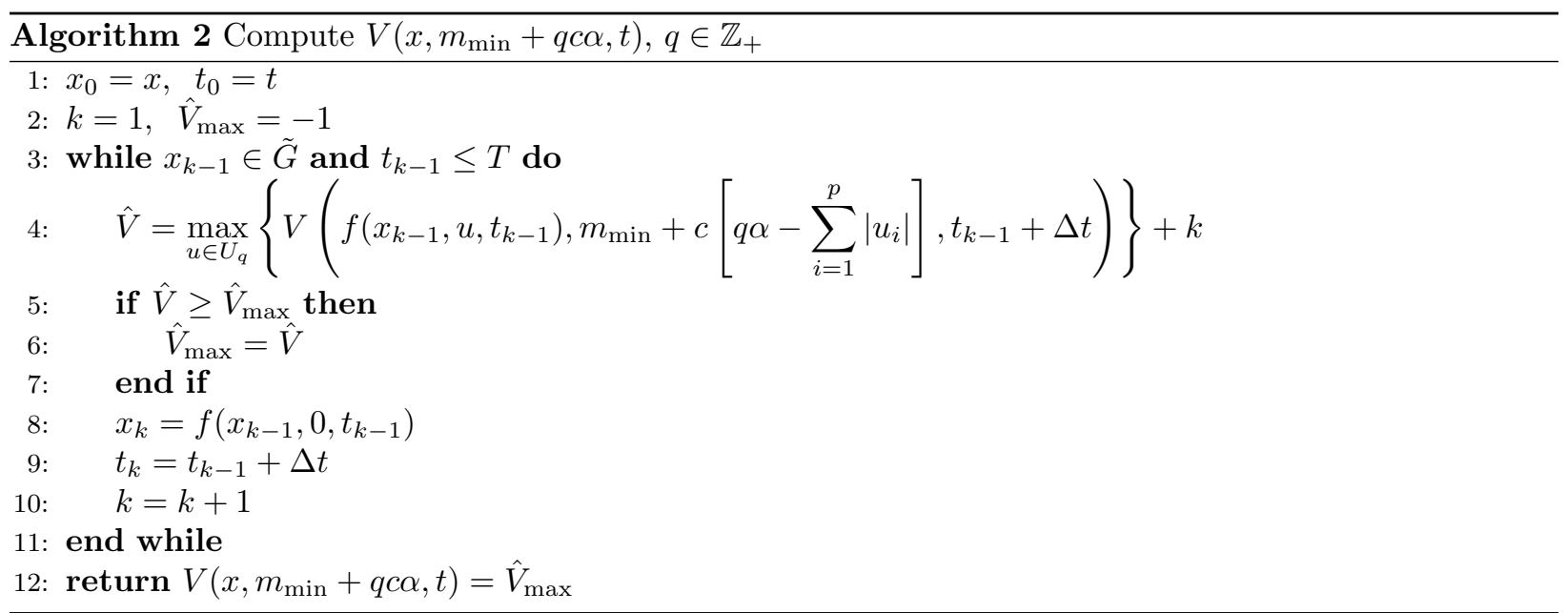

The procedure described by Algorithm 2 is based on recursion, i.e., knowledge of $V\left(x, m_{\min }+(q-1) c \alpha, t\right)$, $V\left(x, m_{\min }+(q-2) c \alpha, t\right), \ldots$, and $V\left(x, m_{\min }, t\right)$ is required to compute $V\left(x, m_{\min }+q c \alpha, t\right)$. In contrast to Algorithm 1 where we initialize $k=0$, Algorithm 2 initializes $k=1$ which takes into account the additional step for switching from the zero-control trajectory to a different trajectory. The function $V$ is computed at the discrete points $x \in \tilde{G}_{\text {dis }}, m \in \mathcal{M}_{\text {dis }}$, and $t \in \mathcal{T}_{\text {dis }}$. We use linear interpolation to evaluate $V$ at $\left\{x \in \tilde{G}: x \notin \tilde{G}_{\text {dis }}\right\}$ and $t \notin \mathcal{T}_{\text {dis }}$. Besides the increased computational efficiency as demonstrated by our ability to handle larger dimensional problems than possible with the conventional value iterations, another advantage of the new method versus the conventional DP algorithms is that convergence is not an issue. This is based on the requirement stated earlier that the system leaves the set $G$ in a finite number of steps for all points in $G$ and $t \geq 0$, i.e., $\tau^{x, m, t, u}(G)$ is finite for any control policy $u$. Consequently, it is guaranteed that there are no infinite loops in Algorithms 1 and 2. In fact, it is possible to exactly determine the total number of computational steps of the procedure.

\section{B. Simplification of the Solution Approach}

The solution approach outlined before can be simplified which also improves the computational performance. We use an assumption that for each initial state $x_{0} \in \tilde{G}$ and time $t_{0} \geq 0$ there exists $q^{*} \in \mathbb{Z}_{+}, u^{\prime} \in U_{q^{*}}$, and 
$k^{\prime} \in \mathbb{Z}_{+}$such that

$$
V\left(x_{0}, m_{\min }+\left(q^{*}+n\right) c \alpha, t_{0}\right)=V\left(f\left(x_{k^{\prime}-1}, u^{\prime}, t_{k^{\prime}-1}\right), m_{\min }+c\left[\left(q^{*}+n\right) \alpha-\sum_{i=1}^{p}\left|u_{i}^{\prime}\right|\right], t_{k^{\prime}-1}+\Delta t\right)+k^{\prime},
$$

for all $n \in \mathbb{Z}_{\geq 0}$. In other words, for a sufficiently high initial mass $m_{0} \geq m_{\min }+q^{*} c \alpha$, the optimal control policy always applies the control action $u^{\prime}=u^{*}\left(x_{k^{\prime}-1}, m_{0}, t_{k^{\prime}-1}\right)$ at the same state $x_{k^{\prime}-1}$ and time $t_{k^{\prime}-1}$ after traversing the zero-control trajectory, starting from $x_{0}$ at time $t_{0}$, for $k^{\prime}-1$ steps. Note that $q^{*}$ as well as $u^{\prime}, k^{\prime}, x_{k^{\prime}-1}$, and $t_{k^{\prime}-1}$ may be functions of $x_{0}$ and $t_{0}$.

In order to explain the underlying idea behind this simplified approach, the set $\mathcal{D} \subset \tilde{G}$ is defined as

$$
\mathcal{D}=\{x \in \tilde{G}: V(x, m, t) \rightarrow \infty \text { for } m \rightarrow \infty \text { and all } t \geq 0\},
$$

The set $\mathcal{D}$ contains all points $x \in \tilde{G}$ for which there exists a control policy $u$ such that the exit time $\tau^{x, m, t, u}(G)$ goes to infinity for the case of unlimited control resources $(m \rightarrow \infty)$. It is intuitive to assume that for all $x \in \mathcal{D}$, if $m \rightarrow \infty$, there exists a trajectory, denoted by $x_{\infty}^{*}=\left\{x_{\infty, k}^{*} \in \tilde{G}, k \in \mathbb{Z}_{\geq 0}\right\}$ with $x=x_{\infty, 0}^{*}$, that requires the least control effort compared to any other trajectory that remains in $\tilde{G}$ for all times. Then, based on Remark 1, for a sufficiently high but finite initial mass it seems advantageous to stay on the trajectory $x_{\infty}^{*}$ for as long as possible. Consequently, for each initial $x_{0} \in \mathcal{D}$ and time $t_{0}$, the optimal control policy always applies the control action $u^{\prime}$ at the same state $x_{k^{\prime}-1}=x_{\infty, k^{\prime}-1}^{*}$ and time $t_{k^{\prime}-1}$ independent of the initial mass $m_{0}$ if $m_{0}$ is sufficiently high. In addition to $x_{0} \in \mathcal{D}$, Eq. (13) also holds for all $x_{0} \in \tilde{G} \cap \mathcal{D}^{\mathrm{C}}$, where $\mathcal{D}^{\mathrm{C}}$ denotes the complement of $\mathcal{D}$. Note that in general $V\left(x_{0}, m_{\min }, t_{0}\right) \leq V\left(x_{0}, m_{\min }+c \alpha, t_{0}\right) \leq$ $V\left(x_{0}, m_{\min }+2 c \alpha, t_{0}\right) \leq V\left(x_{0}, m_{\min }+3 c \alpha, t_{0}\right) \leq \ldots$. Since $x_{0} \in \tilde{G} \cap \mathcal{D}^{\mathrm{C}}$, the exit time $\tau^{x_{0}, m_{0}, t_{0}, u}(G)$ is finite for any control policy $u$. Thus, the sequence $V\left(x_{0}, m_{\min }+\left(q^{*}+n\right) c \alpha, t_{0}\right)$ has to be bounded from above. This and $V$ being integer-valued due to the performance criterion used herein, see Eq. (3), imply that there exists $q^{*} \in \mathbb{Z}_{+}$such that $V\left(x_{0}, m_{\min }+q^{*} c \alpha, t_{0}\right)=V\left(x_{0}, m_{\min }+\left(q^{*}+n\right) c \alpha, t_{0}\right)$ for all $n \in \mathbb{Z}_{\geq 0}$.

An important implication of Eq. (13) is that for $m_{0}=m_{\min }+\left(q^{*}+n\right) c \alpha, n \geq \mathbb{Z}_{+}$, Algorithm 2 becomes unnecessary. By knowing $u^{\prime}$ and $k^{\prime}$ for each $x_{0}$ and $t_{0}$, the function value $V\left(x_{0}, m_{\min }+\left(q^{*}+n\right) c \alpha, t_{0}\right)$ is obtained according to Eq. (13). In contrast, Algorithm 2 has to compute $\hat{V}$ (step 4 in Algorithm 2) at each state on the zero-control trajectory. Thus, by using Eq. (13), the computational time to obtain $V\left(x_{0}, m_{\min }+q c \alpha, t_{0}\right)$ for $q \geq q^{*}$ decreases to a fraction of the computational time of Algorithm 2.

It seems, however, impossible to determine $q^{*}$ for each individual $x_{0}$ and $t_{0}$. For a numerical implementation it is practical to assume a $q^{*}$ which is an upper bound for all $x_{0} \in \tilde{G}_{\text {dis }}$ and $t_{0} \in \mathcal{T}_{\text {dis }}$. For the example in Section 4 we set $q^{*}=8$ for all $x_{0} \in \tilde{G}_{\text {dis }}$ and $t_{0} \in \mathcal{T}_{\text {dis }}$. This value is chosen because, using the simplified approach with Eq. (13), there are no practical differences between the computed value functions for $q^{*} \geq 8$. Moreover, with $q^{*}=8$ for all $x_{0} \in \tilde{G}_{\text {dis }}$ and $t_{0} \in \mathcal{T}_{\text {dis }}$, the value function obtained with Eq. (13) is the same (within numerical accuracy) as the value function obtained by Algorithm 2 and we verified that both value functions satisfy (5) for all $x_{0} \in \tilde{G}_{\text {dis }}$ and $t_{0} \in \mathcal{T}_{\text {dis }}$. Besides the example in Section 4, the simplified method using Eq. (13) was also verified for a two-dimensional mass-spring system with disturbance which is not shown in this paper.

\section{Example: Spacecraft Attitude Control}

\section{A. Model Formulation}

The general rotational dynamics of a rigid body with time-varying mass and inertia properties are derived in the Appendix. Eq. (39) in the Appendix states the governing equation for a rigid body $\mathcal{B}$ with center of mass $c$ using a body-fixed point $z$. The specific spacecraft model for this example is outlined in Fig. 1. A cylindrical spacecraft with four sections is considered. The first section comprises the main engine with evenly distributed mass $m_{\mathrm{e}}$. The engine is followed by the tanks of the oxidizer (ox) and the fuel (f). Both the oxidizer and the fuel have initial masses $m_{\mathrm{ox}, 0}$ and $m_{\mathrm{f}, 0}$, respectively, which decrease at constant mass flow rates $\dot{m}_{\text {ox }}$ and $\dot{m}_{\mathrm{f}}$, respectively. We neglect the slosh dynamics in the tanks. The fourth section of the rocket is the payload with evenly distributed mass $m_{\mathrm{p}}$. 
The governing equations are resolved in the body-fixed principal frame $\mathrm{F}_{\mathrm{B}}$ with mutually perpendicular frame vectors $\left(\hat{b}_{1}, \hat{b}_{2}, \hat{b}_{3}\right)$, where $\hat{b}_{3}$ is the symmetry axis of the spacecraft. As shown in Fig. 1, there is a thrust force $\vec{F}_{\mathrm{t}}$ acting on the spacecraft that is misdirected by an angle $\beta$ relative to the symmetry axis. Moreover, the thrust force is displaced relative to the point $z$ by a radial distance $d_{1}$ and longitudinal distance $d_{2}$. As derived in the Appendix, the equations describing the rotational dynamics of the spacecraft are

$$
\begin{aligned}
& \dot{\omega}_{1}=\frac{u_{1}+M_{1}+\left(2 r_{3}(t) v_{3}(t) m_{\mathcal{B}}(t)+r_{3}^{2}(t) \dot{m}_{\mathcal{B}}-\dot{J}_{\mathrm{T}}(t)\right) \omega_{1}+\left(J_{\mathrm{T}}(t)-J_{\mathrm{R}}(t)-r_{3}^{2}(t) m_{\mathcal{B}}(t)\right) \omega_{2} \omega_{3}-r_{3}(t) f_{2}}{J_{\mathrm{T}}(t)-r_{3}^{2}(t) m_{\mathcal{B}}(t)}, \\
& \dot{\omega}_{2}=\frac{u_{2}+M_{2}+\left(2 r_{3}(t) v_{3}(t) m_{\mathcal{B}}(t)+r_{3}^{2}(t) \dot{m}_{\mathcal{B}}-\dot{J}_{\mathrm{T}}(t)\right) \omega_{2}+\left(J_{\mathrm{R}}(t)-J_{\mathrm{T}}(t)+r_{3}^{2}(t) m_{\mathcal{B}}(t)\right) \omega_{1} \omega_{3}+r_{3}(t) f_{1}}{J_{\mathrm{T}}(t)-r_{3}^{2}(t) m_{\mathcal{B}}(t)},
\end{aligned}
$$

where $\omega_{1}$ and $\omega_{2}$ are the spacecraft's angular velocity vector projections on its body-fixed axes $\hat{b}_{1}$ and $\hat{b}_{2}$, respectively. The angular velocity projection $\omega_{3}=\omega_{3}(t)$ on the symmetry axis $\hat{b}_{3}$ can be obtained explicitly as a function of time, see Eq. (56). The time-dependent parameters $r_{3}(t)$ and $v_{3}(t)$ are the position and velocity of the center of mass $c$ relative to point $z$. The respective equations are given by Eq. (46) and (47). Eq. (41) describes the time-varying mass of the spacecraft $m_{\mathcal{B}}(t)$. The time-varying components of the moment of inertia $J_{\mathrm{R}}(t), J_{\mathrm{T}}(t)$, and its derivative $\dot{J}_{\mathrm{T}}(t)$ are given by Eq. (49), (50), and (52), respectively. The external force acting along the $\hat{b}_{1}$-axis is $f_{1}$ and likewise $f_{2}$ is acting along the $\hat{b}_{2}$-axis. The external moments around the two body-fixed axes are $M_{1}$ and $M_{2}\left(M_{3}=0\right.$ is assumed). There are two control inputs $u_{1} \in\{-\alpha, 0, \alpha\}$ and $u_{2} \in\{-\alpha, 0, \alpha\}$ which are the control moments around the $\hat{b}_{1}$ and $\hat{b}_{2}$-axis, respectively.

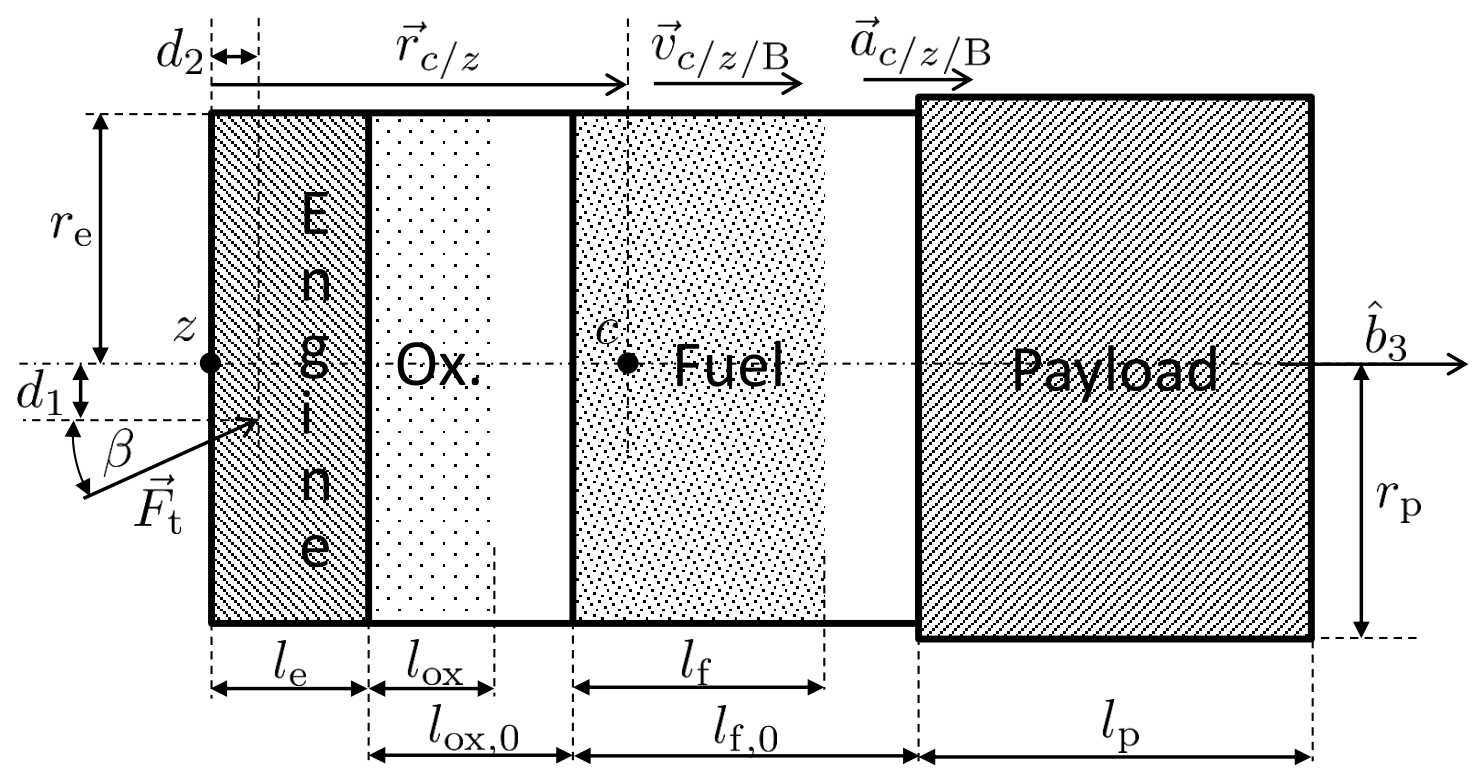

Figure 1. Example axisymmetric spacecraft.

In order to describe the attitude kinematics we use a parametrization introduced by Tsiotras and Longuski. ${ }^{11}$ The orientation of the 3 -axis of the inertial reference frame $\mathrm{F}_{\mathrm{A}}$, denoted by $\hat{a}_{3}$, expressed in the body-fixed frame $\mathrm{F}_{\mathrm{B}}$ is described by the two variables $\theta_{1}$ and $\theta_{2}$

$$
\theta_{1}=\frac{b}{1+c}, \quad \theta_{2}=-\frac{a}{1+c},
$$

where $a, b$, and $c$ are the components of $\hat{a}_{3}$ expressed in the body-fixed frame, i.e., $\hat{a}_{3}=a \hat{b}_{1}+b \hat{b}_{2}+c \hat{b}_{3}$. Note that $\theta_{1}$ is the real part and $\theta_{2}$ is the imaginary part of a complex variable $\theta$ which results from the stereographic projection $\sigma: \mathcal{S}^{2} \backslash\{0,0,-1\} \rightarrow \mathbb{C}$, where $\mathcal{S}^{2}$ denotes the surface of the unit sphere in $\mathbb{R}^{3}$. Using $\theta_{1}$ and $\theta_{2}$ for attitude representation, the kinematic equations are given by ${ }^{11}$ 


$$
\begin{gathered}
\dot{\theta}_{1}=\omega_{3} \theta_{2}+\omega_{2} \theta_{1} \theta_{2}+\frac{\omega_{1}}{2}\left(1+\theta_{1}^{2}-\theta_{2}^{2}\right), \\
\dot{\theta}_{2}=-\omega_{3} \theta_{1}+\omega_{1} \theta_{1} \theta_{2}+\frac{\omega_{2}}{2}\left(1+\theta_{2}^{2}-\theta_{1}^{2}\right) .
\end{gathered}
$$

The available propellant mass for the spacecraft's attitude control system is denoted by $m$. The differential equation describing $m$ is as follows

$$
\dot{m}=-c\left(\left|u_{1}\right|+\left|u_{2}\right|\right)
$$

where $c>0$ is a constant. In summary, the time-dependent system is described by five states: $x=$ $\left[\omega_{1}, \omega_{2}, \theta_{1}, \theta_{2}\right]^{\mathrm{T}}$ and $m$. There are two control inputs $u_{1}$ and $u_{2}$. The governing equations are given by Eq. (15), (16), (18), (19), and (20). The continuous-time model is converted into a discrete-time formulation as stated in Eq. (1) using Euler's forward method

$$
\begin{aligned}
& x_{k+1}=x_{k}+\left[\dot{\omega}_{1}\left(t_{k}\right), \dot{\omega}_{2}\left(t_{k}\right), \dot{\theta}_{1}\left(t_{k}\right), \dot{\theta}_{2}\left(t_{k}\right)\right]^{\mathrm{T}} \Delta t, \\
& m_{k+1}=m_{k}+\dot{m}\left(t_{k}\right) \Delta t,
\end{aligned}
$$

where a sampling time of $\Delta t=0.3 \mathrm{sec}$ is used for this example.

\section{B. Model Parameters}

The engine characteristics of the spacecraft are similar to the A-4 engine ${ }^{12}$ which uses Hydrazine as fuel and Dinitrogen Tetroxide as the oxidizer. The engine generates a thrust of $F_{\mathrm{t}}=33,360 \mathrm{~N}$ with $I_{\mathrm{sp}}=320$ sec and oxidizer and fuel mass flow rates of $\dot{m}_{\mathrm{ox}}=5.8 \mathrm{~kg} / \mathrm{sec}$ and $\dot{m}_{\mathrm{f}}=4.83 \mathrm{~kg} / \mathrm{sec}$, respectively. ${ }^{2}$ With an engine mass of $m_{\mathrm{e}}=117 \mathrm{~kg}$, initial fuel and oxidizer masses of $m_{\mathrm{f}, 0}=2273 \mathrm{~kg}$ and $m_{\mathrm{ox}, 0}=2727 \mathrm{~kg}$, and a payload mass of $m_{\mathrm{p}}=8,000 \mathrm{~kg}$, the total wet mass of the spacecraft is $m_{\mathcal{B}, 0}=13,117 \mathrm{~kg}$. The burn time for the orbital maneuver is $T=200 \mathrm{sec}$. Note that this maneuver generates an increase in velocity of $\Delta v=0.56 \mathrm{~km} / \mathrm{sec}$. For this example, a nominal thrust vector misalignment of $\beta=0.1 \mathrm{deg}$ and $d_{1}=2 \mathrm{~mm}$ are assumed. Moreover, $d_{2}=1 \mathrm{~m}$ is chosen according to Fig. 1 . Therefore, the components of the external moment relative to point $z$ are

$$
M_{1}=d_{1} F_{\mathrm{t}} \cos (\beta)=66.72 \mathrm{Nm}, \quad M_{2}=d_{2} F_{\mathrm{t}} \sin (\beta)=58.22 \mathrm{Nm}, \quad M_{3}=0 .
$$

The components of the external force acting on the spacecraft are

$$
f_{1}=-F_{\mathrm{t}} \sin (\beta)=-58.22 \mathrm{~N}, \quad f_{2}=0 \mathrm{Nm}, \quad f_{3}=F_{\mathrm{t}} \cos (\beta)=33,359.9 \mathrm{~N} .
$$

The attitude control system for the axes $\hat{b}_{1}$ and $\hat{b}_{2}$ comprises eight R-4D thrusters. ${ }^{13}$ Each thruster can generate a force of $490 \mathrm{~N}$ with an $I_{\mathrm{sp}}$ of $312 \mathrm{sec}$. With an effective lever of $2.86 \mathrm{~m}$, each pair of R- $4 \mathrm{D}$ thrusters generates a moment of $1,401 \mathrm{Nm}$ about the respective axis $\left(\hat{b}_{1}\right.$ or $\left.\hat{b}_{2}\right)$ relative to point $z$. Thus, the control inputs $u_{1}$ and $u_{2}$ take values from the set $\{-1401,0,1401\} \mathrm{Nm}$. The constant $c$ in Eq. (20) that describes the propellant consumption of the attitude control system is $c=2 / I_{\mathrm{sp}} / g / 2.86 \mathrm{~m}=2.285 \times 10^{-4} \mathrm{sec} / \mathrm{m}^{2}$. The remaining parameters of the spacecraft are summarized in the following table.

Table 1. Parameters of the example spacecraft.

\begin{tabular}{c|c|c|c}
$l_{\mathrm{p}}=3 \mathrm{~m}$ & $l_{\mathrm{ox}, 0}=0.96 \mathrm{~m}$ & $l_{\mathrm{e}}=1.75 \mathrm{~m}$ & $l_{\mathrm{f}, 0}=1.15 \mathrm{~m}$ \\
\hline$r_{\mathrm{e}}=0.8 \mathrm{~m}$ & $r_{\mathrm{p}}=1.5 \mathrm{~m}$ & $\rho_{\mathrm{ox}}=1456 \mathrm{~kg} / \mathrm{m}^{3}$ & $\rho_{\mathrm{f}}=1013 \mathrm{~kg} / \mathrm{m}^{3}$
\end{tabular}

\section{Set $G$ and State Space Discretization}

The allowed set $G$ is given by

$$
G=\left\{(x, m) \in \mathbb{R}^{4} \times \mathbb{R}: \sqrt{\theta_{1}^{2}+\theta_{2}^{2}} \leq \theta_{\text {limit }}, m \geq 0\right\} .
$$


The first part of $G$, referred to as $\tilde{G}$ thus far, describes the allowed orientation of the spacecraft's symmetry axis. It defines a cone, where a half angle of $0.5 \mathrm{deg}$ is chosen here. This corresponds to $\theta_{\text {limit }}=0.004363$ according to Eq. (17). The minimum propellant mass for the attitude control system is $m_{\min }=0$.

In order to discretize the state and time space for the numerical simulations the following function is defined which is similar to Matlab's linspace

$$
\operatorname{grid}\left(x_{0}, x_{1}, n\right)=\left\{x_{0}, x_{0}+\frac{x_{1}-x_{0}}{n-1}, x_{0}+2 \frac{x_{1}-x_{0}}{n-1}, \ldots, x_{1}\right\}, \quad x_{0}, x_{1} \in \mathbb{R}, \quad x_{0}<x_{1}, \quad n \in \mathbb{Z}_{+} .
$$

This function creates a grid of $n$ points with equidistant spacing. The following nominal discretization is used for this example

$$
\begin{gathered}
\tilde{G}_{\text {dis }}=\left\{x \in \tilde{G}: \omega_{1}, \omega_{2} \in \operatorname{grid}(-0.86 \mathrm{deg} / \mathrm{sec}, 0.86 \mathrm{deg} / \mathrm{sec}, 29), \theta_{1}, \theta_{2} \in \operatorname{grid}(-0.004363,0.004363,17)\right\}, \\
\mathcal{T}_{\text {dis }}=\operatorname{grid}(0,200 \mathrm{sec}, 40), \\
\mathcal{M}_{\text {dis }}=\operatorname{grid}(0,15.27 \mathrm{~kg}, 160) .
\end{gathered}
$$

The chosen grid for $m$ provides enough resources to feed the attitude control system during the 200 sec maneuver. Besides the solution for the nominal discretization, we also investigate the influence of different discretization choices on the solution in the next section.

\section{Results}

Algorithms 1 and 2 are used to compute $V$ at the points of the discretized state and time spaces given by Eq. (26). With $q^{*}=8$, we use the simplified approach from Section 3-B and Eq. (13) instead of Algorithm 2 if $m \geq 0.77 \mathrm{~kg}$. Note that $V=0$ at all points that do not satisfy $x \in \tilde{G}$. Therefore, those points are not considered by Algorithms 1 and 2. Moreover, we initially check if for each grid point $\left(x_{0}, m_{0}, t_{0}\right)$ there exists a control $u_{0} \in U$ such that $\left(x_{1}\left(u_{0}\right), m_{1}\left(u_{0}\right)\right) \in G$. If not we set $V=1$ and the point is not considered by Algorithm 1 and 2. The method is implemented as a C program. All simulations were run on a desktop computer with an Intel Core i7-3770 processor and 15.8 GB usable memory.

According to Theorem 1 and (5), the value function $V$ has to decrease by one at each time instant when using the optimal control policy. Since the sampling time is $\Delta t=0.3 \mathrm{sec}$, the slope of $V$ versus the time is $-10 / 3$ when using the optimal control policy. Therefore, the following criterion is introduced to asses the difference of the numerical solution to the optimal solution

$$
\Delta V / \Delta t_{0.3}=0.3\left(\frac{V\left(x_{\tau-1}, m_{\tau-1}, t_{\tau-1}\right)-1-V\left(x_{0}, m_{0}, t_{0}\right)}{t_{\tau}}\right)=-0.3 \frac{V\left(x_{0}, m_{0}, t_{0}\right)}{t_{\tau}},
$$

where $\tau=\tau^{x_{0}, m_{0}, t_{0}, u}(G)$ is the time instant at which the system leaves the set $G$ for the first time and $\Delta V / \Delta t_{0.3}=-1$ for the optimal solution. We stress that $\Delta V / \Delta t_{0.3}=-1$ is a necessary condition for optimality. A computational time of $439 \mathrm{sec}$ was required to solve the DDCOC problem numerically and compute $V$ on the nominal grid $\tilde{G}_{\text {dis }} \times \mathcal{T}_{\text {dis }} \times \mathcal{M}_{\text {dis. }}$. Fig. 2 shows the simulation results for an initial condition of $\omega_{0,1}=\omega_{0,2}=\theta_{0,1}=\theta_{0,2}=0, m_{0}=15.27 \mathrm{~kg}$, and $t_{0}=0$. The spacecraft stays inside the prescribed set $G$ during the entire maneuver time of $200 \mathrm{sec}$. This can be seen in Fig. 2 (c) which shows the trajectory of the attitude parameters in the complex plane including the boundary $\theta_{\text {limit }}$ (red circle) and in Fig. 2 (d) which shows the propellant mass. The remaining propellant mass at the end is $0.67239 \mathrm{~kg}$.

The cost-to-go function is linearly decreasing in time as seen in Fig. 2 (f). The criterion from Eq. (27) is $\Delta V / \Delta t_{0.3}=-0.9954$ which suggests that the numerical solution is as close as $0.46 \%$ to being optimal (based on necessary conditions). The control input is plotted in Fig. 2 (e). Both $u_{1}$ and $u_{2}$ show a similar pattern, where a short non-zero control pulse is followed by a longer coasting period. The pulses of $u_{2}$ are more frequent because the disturbance moment (due to $f_{1}$ ) about the $\hat{b}_{2}$-axis is larger than the disturbance about the $\hat{b}_{1}$-axis. The control moments are mainly applied in the direction opposite to the external disturbance. However, there are a few deviations from this direction for both $u_{1}$ and $u_{2}$. This may be due to inaccuracies caused by linear interpolation which is required to compute $V$ between the grid points. Furthermore, this may be the reason why the numerical solution is $0.46 \%$ away from satisfying the necessary condition for optimality, $\Delta V / \Delta t_{0.3}=-1$. A denser grid can slightly improve the result as shown below. However, a denser grid also increases the computational time. Therefore, the nominal grid provides a good compromise for the trade-off between computational performance and accuracy of the solution. 

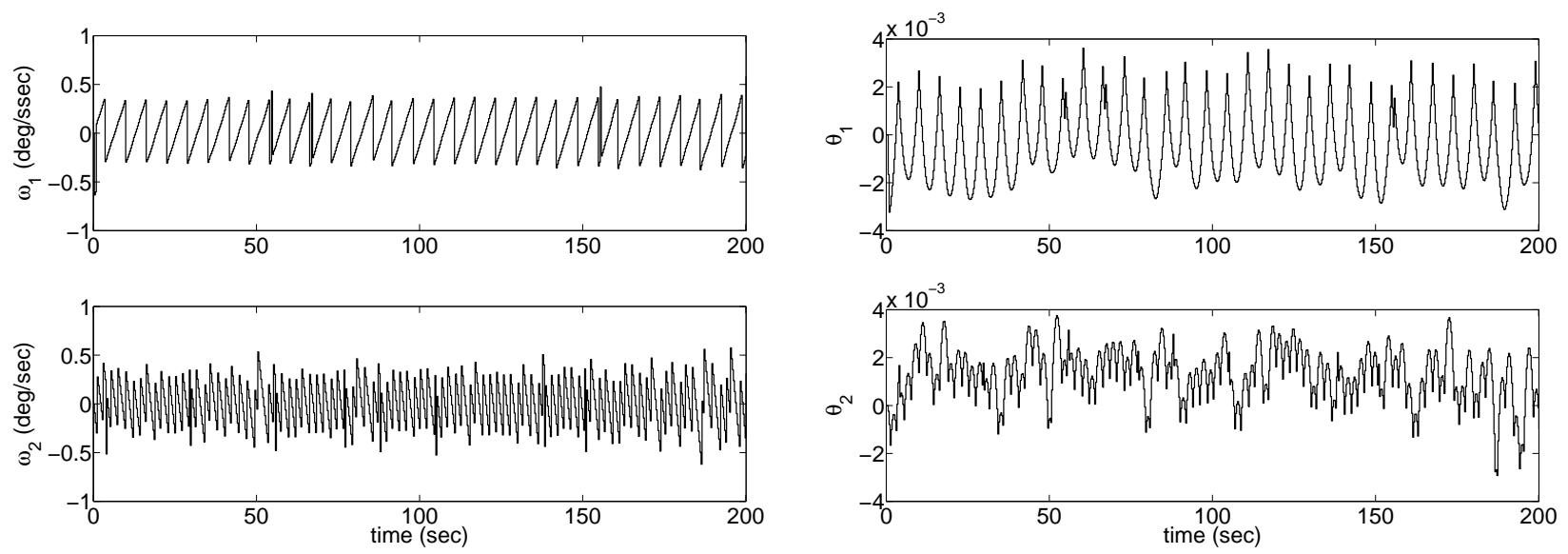

(a) Angular velocities $\omega_{1}$ and $\omega_{2}$ vs. time

(b) Attitude parameters $\theta_{1}$ and $\theta_{2}$ vs. time
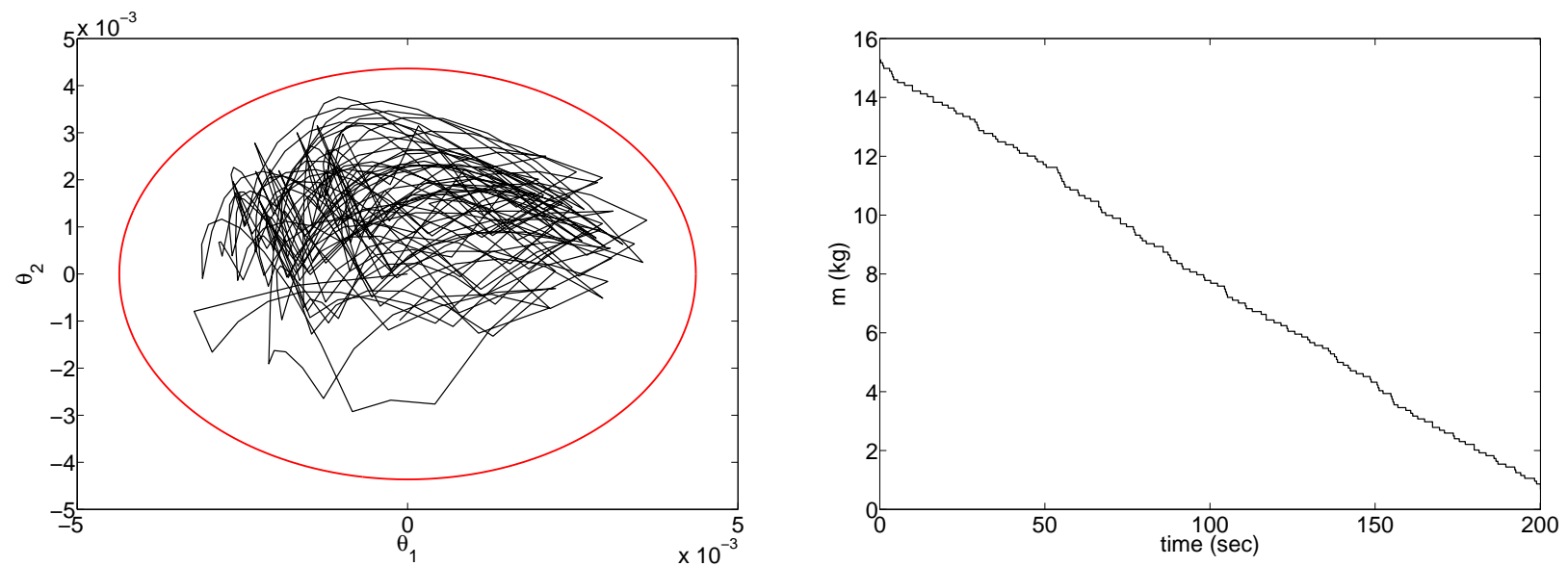

(c) Attitude parameters in complex plane vs. boundary $\theta_{\text {limit }}$

(d) Mass $m$ vs. time
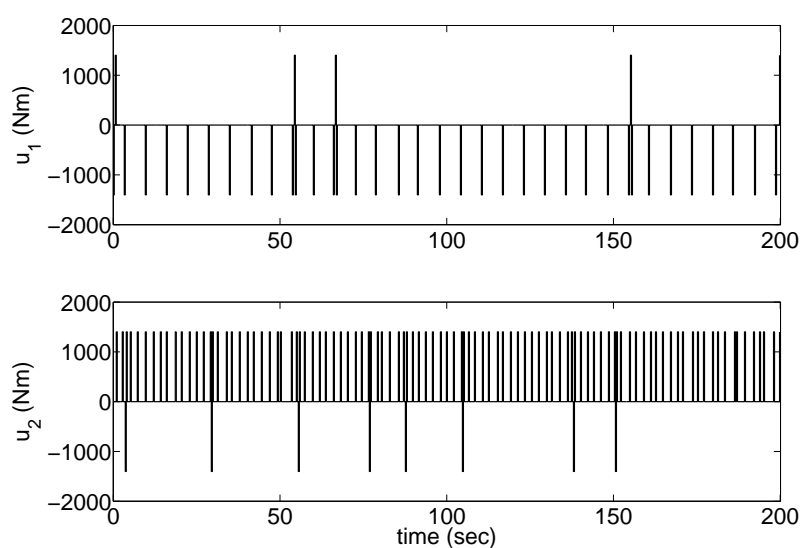

(e) Control moments $u_{1}$ and $u_{2}$ vs. time

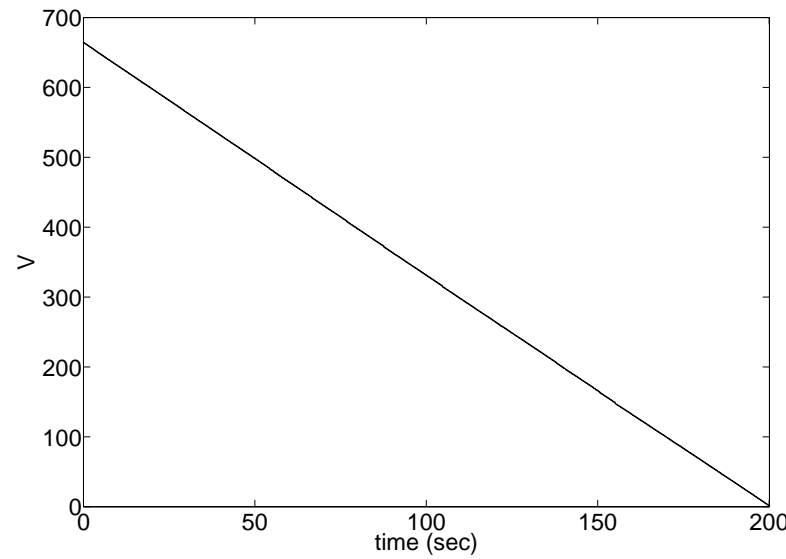

(f) Cost-to-go function $V$ vs. time

Figure 2. Example spacecraft attitude control for nominal disturbance, Eq.(22) and (23), nominal grid, Eq. (26), and initial condition $\omega_{0,1}=\omega_{0,2}=\theta_{0,1}=\theta_{0,2}=0, m_{0}=15.27 \mathrm{~kg}$. 

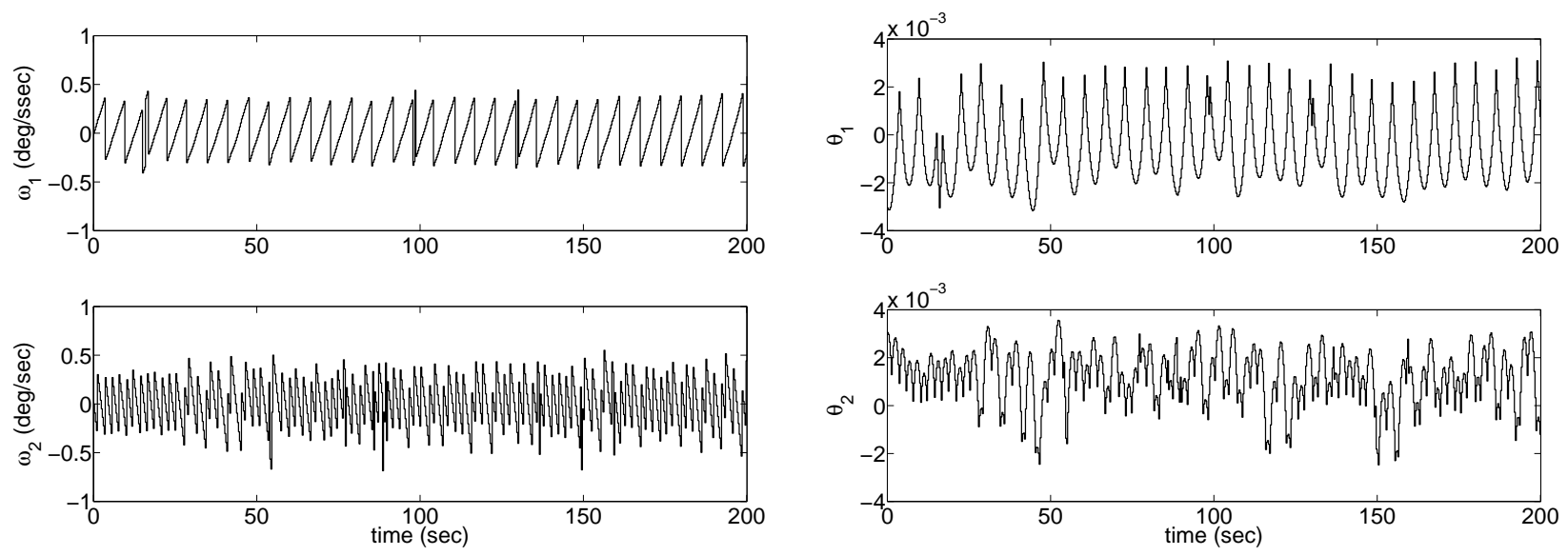

(a) Angular velocities $\omega_{1}$ and $\omega_{2}$ vs. time

(b) Attitude parameters $\theta_{1}$ and $\theta_{2}$ vs. time
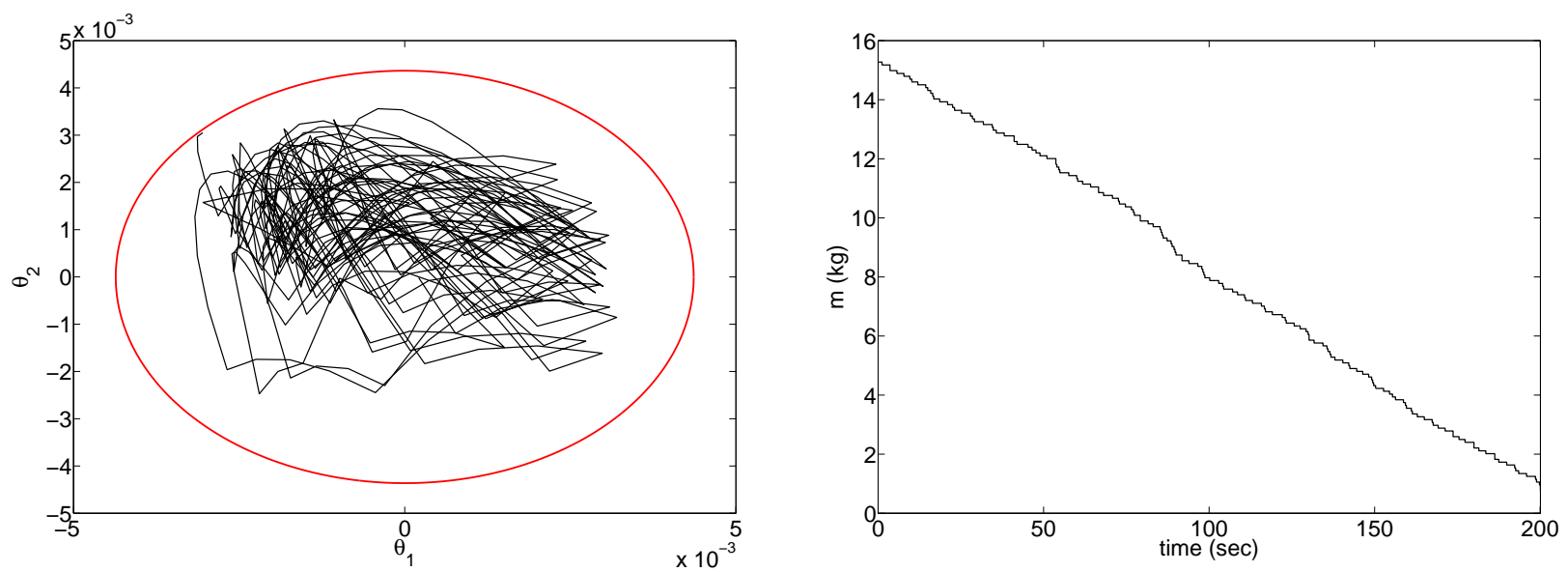

(c) Attitude parameters in complex plane vs. boundary $\theta_{\text {limit }}$

(d) Mass $m$ vs. time
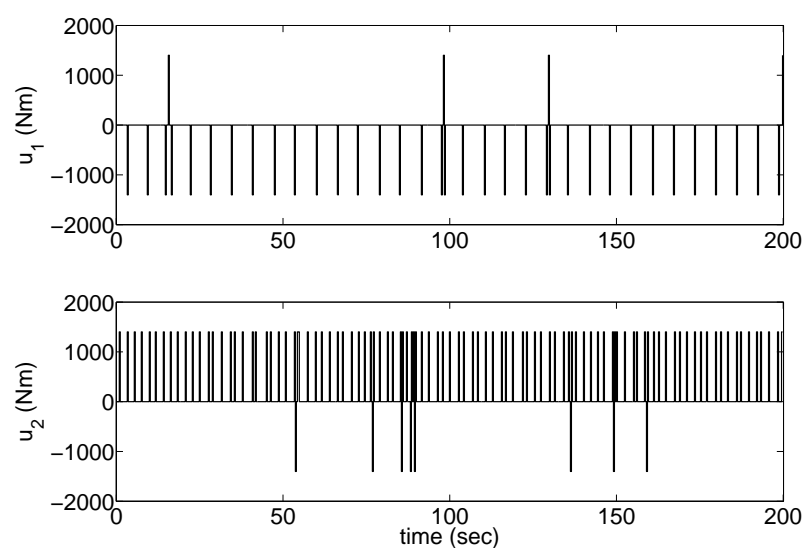

(e) Control moments $u_{1}$ and $u_{2}$ vs. time

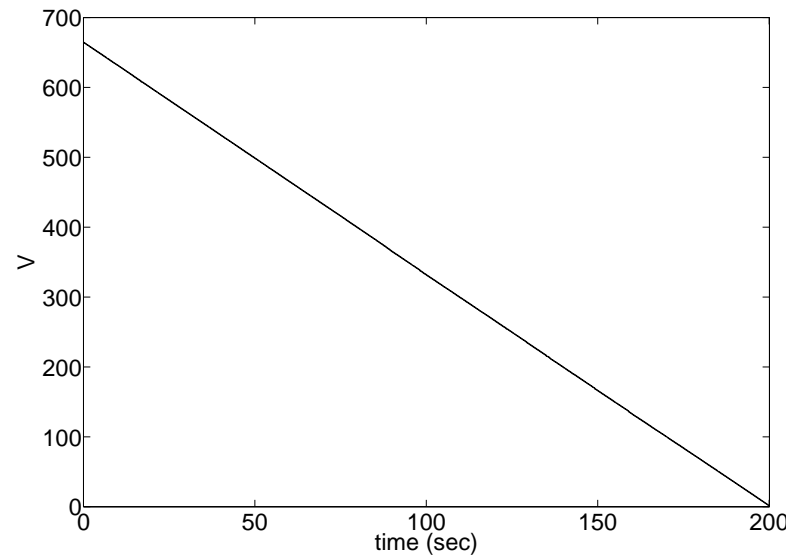

(f) Cost-to-go function $V$ vs. time

Figure 3. Example spacecraft attitude control for nominal disturbance, Eq. (22) and (23), nominal grid, Eq. (26), and initial condition $\omega_{0,1}=\omega_{0,2}=0, \theta_{0,1}=-0.00305, \theta_{0,2}=0.00305, m_{0}=15.27 \mathrm{~kg}$.

Fig. 3 shows the simulation results for a different initial condition (using the same value function $V$ as before). Instead of $\theta_{0,1}=\theta_{0,2}=0$, it is now $\theta_{0,1}=-0.00305$ and $\theta_{0,2}=0.00305$ which is close to the boundary of the allowed cone. By comparing Fig. 3 with Fig. 2 it can be seen that after an initial reorientation the system behaves nearly identical to the case where $\theta_{0,1}=\theta_{0,2}=0$. This suggests that, 
provided there is sufficient propellant mass, the DDCOC law steers the system from any initial condition to a certain region in the state space where it is most efficient to stay. In this case the remaining propellant mass for the attitude control system is $0.7684 \mathrm{~kg}$. The slope of the cost-to-go function versus the time is again close to satisfying the necessary condition for optimality, where $\Delta V / \Delta t_{0.3}=-0.9958$.

Note that for both initial condition cases the initial value of the cost-to-go function is approximately $V(t=0)=667$ which means that $t_{\tau^{x_{0}, m_{0}, t_{0}, u}(G)}=200.1 \mathrm{sec}$ even though there is propellant left at the end. This is due to the discretization of the time with a maximum time of $200 \mathrm{sec}$ as shown by Eq. (26). For numerical reasons we set $V=0$ for all points with $t>200 \mathrm{sec}$. Thus, increasing the maximum time in $\mathcal{T}_{\text {dis }}$ would increase $V(t=0)$ which, however, would not significantly affect $m(t=200 \mathrm{sec})$ and the simulation results for the example $200 \mathrm{sec}$ maneuver.

The influence of the state and time space discretization is investigated by defining two additional discretizations. In contrast to the nominal discretization in Eq. (26) with $n_{\omega}=29$ grid points for $\omega_{1}$ and $\omega_{2}$, $n_{\theta}=17$ grid points for $\theta_{1}$ and $\theta_{2}$, and $n_{t}=40$ grid points for the time, we define a dense discretization with $n_{\omega}=32, n_{\theta}=20$, and $n_{t}=45$ as well as a sparse discretization with $n_{\omega}=25, n_{\theta}=14$, and $n_{t}=36$. Table 2 compares the remaining mass at $t=200 \mathrm{sec}$, the criterion $\Delta V / \Delta t_{0.3}$, and the computational time for the three discretizations sparse, nominal, and dense. The computational time increases exponentially with the density of the discretization. The sparse grid requires $183 \mathrm{sec}$ of computational time in contrast to the dense grid with 909 sec. However, the accuracy of the solution improves with the grid density. The sparse grid generates a solution which is $1.4 \%$ away from being optimal, whereas the solution of the dense grid is as close as $0.12 \%$ to being optimal (based on necessary conditions). This is also reflected in the remaining mass after $200 \mathrm{sec}$. The solution of the sparse grid has no propellant left after $200 \mathrm{sec}$ (however not violating the constraints). Both the solutions for the nominal and the dense grid have $0.67239 \mathrm{~kg}$ propellant left at the end which is because they are closer to the optimal solution than the sparse grid solution (compare $\Delta V / \Delta t_{0.3}$ ).

Table 2. Influence of state and time space discretization on the simulation results for nominal disturbance, Eq.(22) and (23), and an initial condition of $\omega_{0,1}=\omega_{0,2}=\theta_{0,1}=\theta_{0,2}=0, m_{0}=15.27 \mathrm{~kg}$.

\begin{tabular}{cccc} 
Discretization & $m(t=200 \mathrm{sec})$ & $\Delta V / \Delta t_{0.3}$ & Computational time \\
\hline sparse $n_{\omega}=25, n_{\theta}=14, n_{t}=36$ & $0.0 \mathrm{~kg}$ & -0.986 & $183 \mathrm{sec}$ \\
nominal $n_{\omega}=29, n_{\theta}=17, n_{t}=40$ & $0.67239 \mathrm{~kg}$ & -0.9954 & $439 \mathrm{sec}$ \\
dense $n_{\omega}=32, n_{\theta}=20, n_{t}=45$ & $0.67239 \mathrm{~kg}$ & -0.9988 & $909 \mathrm{sec}$
\end{tabular}

\section{E. Robustness Analysis}

The results in the previous section were obtained with exact knowledge of the disturbances $f_{1}, f_{2}, M_{1}$, and $M_{2}$. In this section the robustness of the solution is analyzed with respect to uncertainties in the disturbances. We use the nominal cost-to-go function obtained for the disturbance values in Eq. (22) and (23) and the discretization in Eq. (26). However, the actual values of the disturbances are increased/decreased from the nominal case by $10 \%, 25 \%$, and $50 \%$.

Table 3 shows the differences in the solution for the initial condition case $\omega_{0,1}=\omega_{0,2}=\theta_{0,1}=\theta_{0,2}=0$, $m_{0}=15.27$. It can be seen that there are no significant differences in the solutions for a $10 \%$ uncertainty in the disturbances. The solutions for the $10 \%$ uncertainty case have the same $\Delta V / \Delta t_{0.3}=-0.9954$ as the nominal solution. For a $25 \%$ uncertainty, however, the solutions start to deviate from the nominal solution. Increasing the disturbances by $25 \%$ from the nominal values results in $\Delta V / \Delta t_{0.3}=-1.031$ which is $3.1 \%$ away from being optimal. Moreover, the constraints are violated before the end of the 200 sec maneuver. Likewise, a $25 \%$ increase from the nominal disturbance values yields $\Delta V / \Delta t_{0.3}=-1.0742$ and constraint violation at $185.4 \mathrm{sec}$. An uncertainty of $50 \%$ in the disturbances results in $\Delta V / \Delta t_{0.3}=-1.2186(21.86 \%$ away from being optimal) and $\Delta V / \Delta t_{0.3}=-1.3025$ (30.25\% away from being optimal), respectively.

An interesting case is when the actual disturbances are zero $\left(f_{1}=f_{2}=M_{1}=M_{2}=0\right)$. For this case and the particular initial condition it would be intuitive not to apply any control action. However, the DDCOC law is based on the nominal disturbance values and tries to steer the system to a supposedly more efficient region. This is certainly not optimal in this case and the constraints are violated after $145.8 \mathrm{sec}$ with $\Delta V / \Delta t_{0.3}=-1.3647$. 
Table 3. Robustness analysis of the DDCOC law with respect to uncertainties in the disturbances for an initial condition of $\omega_{0,1}=\omega_{0,2}=\theta_{0,1}=\theta_{0,2}=0, m_{0}=15.27 \mathrm{~kg}$. The DDCOC law for the simulations is based on the nominal $V$.

\begin{tabular}{cccc} 
Disturbances & $t_{\tau^{x_{0}, m_{0}, t_{0}, u}(G)}$ & $m\left(t_{\tau^{x_{0}, m_{0}, t_{0}, u}(G)}\right) \mathrm{or} m(t=200 \mathrm{sec})$ & $\Delta V / \Delta t_{0.3}$ \\
\hline nominal & $>200 \mathrm{sec}$ & $0.67239 \mathrm{~kg}$ & -0.9954 \\
$10 \%$ increase from nominal & $>200 \mathrm{sec}$ & $0.76845 \mathrm{~kg}$ & -0.9954 \\
$10 \%$ decrease from nominal & $>200 \mathrm{sec}$ & $0.76845 \mathrm{~kg}$ & -0.9954 \\
$25 \%$ increase from nominal & $193.2 \mathrm{sec}$ & $0.0 \mathrm{~kg}$ & -1.031 \\
$25 \%$ decrease from nominal & $185.4 \mathrm{sec}$ & $0.0 \mathrm{~kg}$ & -1.0742 \\
$50 \%$ increase from nominal & $163.5 \mathrm{sec}$ & $0.0 \mathrm{~kg}$ & -1.2186 \\
$50 \%$ decrease from nominal & $153 \mathrm{sec}$ & $0.0 \mathrm{~kg}$ & -1.3025 \\
zero disturbances & $145.8 \mathrm{sec}$ & $0.0 \mathrm{~kg}$ & -1.3647
\end{tabular}

\section{F. Example Control Surface Plots}

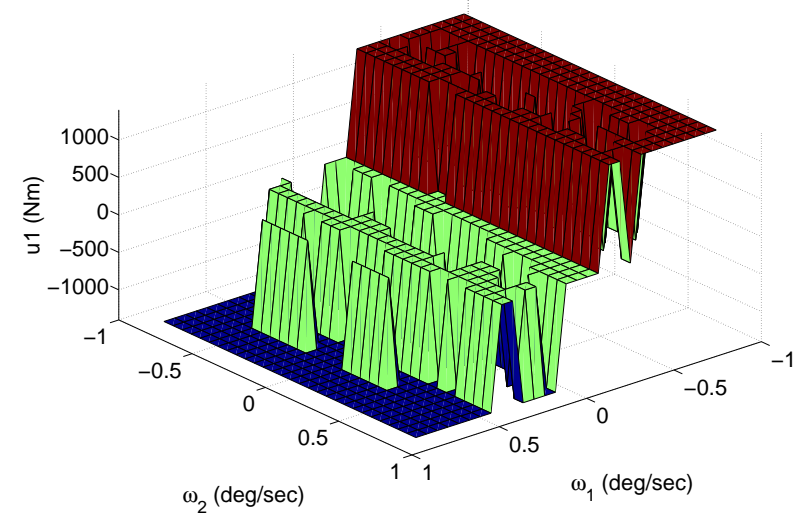

(a) $u_{1}$ vs. $\omega_{1}$ and $\omega_{2}$ for $\theta_{1}=\theta_{2}=0, m=15.27 \mathrm{~kg}, t=0$ fixed.

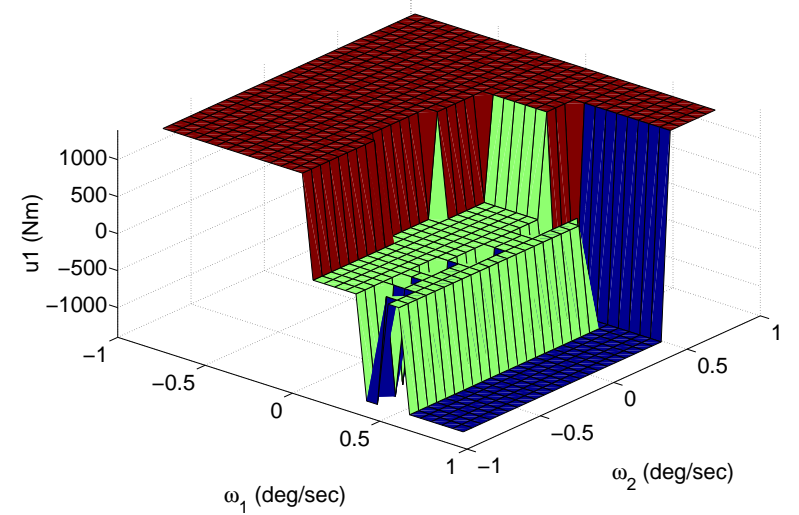

(c) $u_{1}$ vs. $\omega_{1}$ and $\omega_{2}$ for $\theta_{1}=-0.00305, \theta_{2}=0.00305, m=$ $15.27 \mathrm{~kg}, t=100 \mathrm{sec}$ fixed.

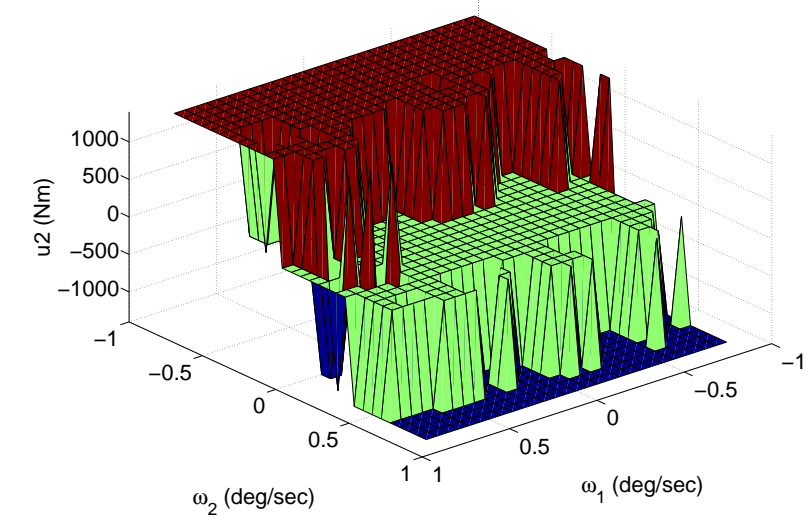

(b) $u_{2}$ vs. $\omega_{1}$ and $\omega_{2}$ for $\theta_{1}=\theta_{2}=0, m=15.27 \mathrm{~kg}, t=0$ fixed.

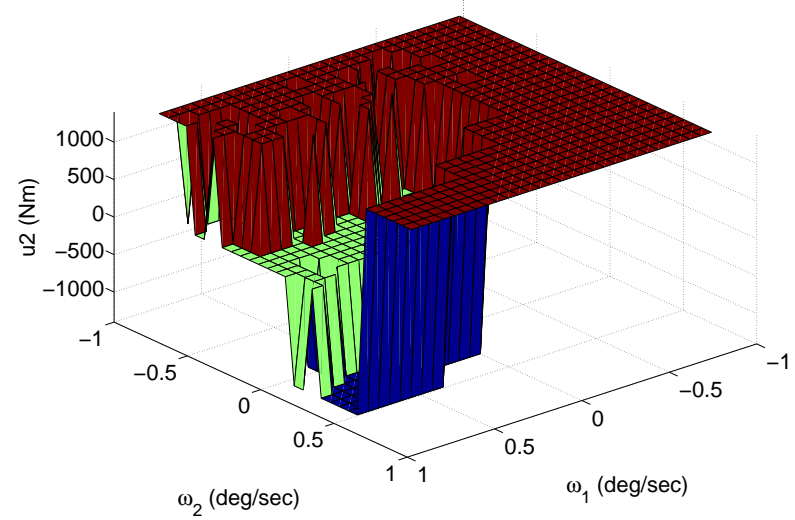

(d) $u_{2}$ vs. $\omega_{1}$ and $\omega_{2}$ for $\theta_{1}=-0.00305, \theta_{2}=0.00305, m=$ $15.27 \mathrm{~kg}, t=100 \mathrm{sec}$ fixed.

Figure 4. Example control surface plots.

Fig. 4 shows example control surface plots of $u_{1}$ and $u_{2}$ versus $\omega_{1}$ and $\omega_{2}$ for two different settings of $\theta_{1}, \theta_{2}$, $m$, and $t$. Fig. 4 (a) and (b) visualize $u_{1}$ and $u_{2}$ for a fixed $\theta_{1}=\theta_{2}=0, m=15.27 \mathrm{~kg}$, and $t=0$. The general trend is as expected, i.e., if $\omega_{1}<0$ then $u_{1}>0$ and vice versa as well as if $\omega_{2}<0$ then $u_{2}>0$ and 
vice versa. However, there are several deviations from this trend that are not immediately intuitive. Fig. 4 (c) and (d) visualize $u_{1}$ and $u_{2}$ for a fixed $\theta_{1}=-0.00305, \theta_{2}=0.00305, m=15.27 \mathrm{~kg}$, and $t=100 \mathrm{sec}$. In contrast to the previous case, the spacecraft's symmetry axis is not in the center of the allowed cone. Thus, a general trend as in Fig. 4 (a) and (b) can not be observed.

\section{Conclusion and Future Work}

A novel approach to solve deterministic drift counteraction optimal control (DDCOC) problems for a special class of nonlinear systems was presented. In contrast to previous work which used conventional dynamic programming algorithms to solve the DDCOC problem, the method is computationally more efficient and allows for higher-dimensional systems. Moreover, obtaining the optimal solution is guaranteed since convergence is not an issue. The approach was applied to an attitude control problem for an axisymmetric spacecraft with thrust vector misalignment imposing substantial disturbances on the system. The spacecraft was modeled with five states, two control inputs, and time-varying mass and inertia properties due to the main engine's propellant flow. The objective for the DDCOC policy was to control the spacecraft such that its symmetry axis stays within a prescribed cone for as long as possible given limited amount of fuel for the attitude control system. It was shown that the proposed method could successfully solve the DDCOC problem. Moreover, the DDCOC policy appeared to be robust against uncertainties in the disturbances of up to $25 \%$. In addition, the trade-off between solution accuracy and computational time due to the state and time space discretization was shown.

Future work will focus on computationally more efficient discretizations of the state and time space. Furthermore, the method will be extended to drift counteraction optimal control for stochastic systems.

\section{Appendix. Rotational Dynamics of a Rigid Body with Time-Varying Mass/Inertia Properties}

The notations for the derivation in this section are adopted from. ${ }^{14}$ The position of a point $x$ relative to a point $y$ is described by the physical vector $\vec{r}_{x / y}$. The physical vector $\vec{r}_{x / y}$ resolved in the frame $\mathrm{F}_{\mathrm{A}}$ is denoted by $\left.\vec{r}_{x / y}\right|_{\mathrm{A}}$. The time derivative of $\vec{r}_{x / y}$ with respect to the frame $\mathrm{F}_{\mathrm{A}}$ is denoted by $\stackrel{\vec{r}_{x / y}}{\text {. The }}$ velocity of a point $x$ relative to a point $y$ with respect to the frame $\mathrm{F}_{\mathrm{A}}$ is $\vec{v}_{x / y / \mathrm{A}}=\stackrel{\mathrm{A}}{\vec{r}}_{x / y}$. Likewise, the acceleration of a point $x$ relative to a point $y$ with respect to the frame $\mathrm{F}_{\mathrm{A}}$ is $\vec{a}_{x / y / \mathrm{A}}=\stackrel{\mathrm{A} \bullet}{\vec{r}_{x / y}}$.

Let $\mathcal{B}$ be a rigid body and $w$ and $z$ are points. Then the following relation holds between the moment on $\mathcal{B}$ relative to $z$ and the moment on $\mathcal{B}$ relative to $w$

$$
\vec{M}_{\mathcal{B} / z}=\vec{M}_{\mathcal{B} / w}-\vec{r}_{z / w} \times \vec{f}_{\mathcal{B}}
$$

where $\overrightarrow{\mathcal{H}}_{\mathcal{B}}$ denotes the total force acting on $\mathcal{B}$. Now $w$ is assumed to be an unforced particle (a particle that has no force applied on $\left.i t^{14}\right)$. Then

$$
\vec{M}_{\mathcal{B} / w}=\stackrel{\stackrel{\text { A }}{\vec{H}}}{\mathcal{B} / w / \mathrm{A}},
$$

where $\vec{H}_{\mathcal{B} / w / \mathrm{A}}$ denotes the angular momentum of $\mathcal{B}$ relative to $w$ with respect to the frame $\mathrm{F}_{\mathrm{A}}$. Substituting Eq. (28) into Eq. (29) yields

$$
\vec{M}_{\mathcal{B} / z}=\stackrel{\text { A }}{\vec{H}_{\mathcal{B} / w / \mathrm{A}}}-\vec{r}_{z / w} \times \vec{f}_{\mathcal{B}} .
$$

By denoting the center of mass of the body $\mathcal{B}$ by $c$, it is straightforward to show that

$$
\vec{H}_{\mathcal{B} / w / \mathrm{A}}=\vec{H}_{\mathcal{B} / z / \mathrm{A}}+\vec{r}_{c / z} \times m_{\mathcal{B}} \vec{v}_{z / w / \mathrm{A}}+\vec{r}_{z / w} \times m_{\mathcal{B}} \vec{v}_{c / w / \mathrm{A}}
$$

where $m_{\mathcal{B}}$ is the time-varying total mass of the body $\mathcal{B}$. The time derivative of Eq. (31) with respect to frame $\mathrm{F}_{\mathrm{A}}$ yields

$$
\begin{aligned}
\mathrm{A}_{\vec{H} / w / \mathrm{A}}= & \stackrel{\vec{H}}{\mathcal{H} / z / \mathrm{A}}+m_{\mathcal{B}}\left(\vec{v}_{c / z / \mathrm{A}} \times \vec{v}_{z / w / \mathrm{A}}+\vec{v}_{z / w / \mathrm{A}} \times \vec{v}_{c / w / \mathrm{A}}+\vec{r}_{z / w} \times \vec{a}_{c / w / \mathrm{A}}+\vec{r}_{c / z} \times \vec{a}_{z / w / \mathrm{A}}\right) \\
& +\dot{m}_{\mathcal{B}}\left(\vec{r}_{c / z} \times \vec{v}_{z / w / \mathrm{A}}+\vec{r}_{z / w} \times \vec{v}_{c / w / \mathrm{A}}\right),
\end{aligned}
$$


with $\dot{m}_{\mathcal{B}}=\mathrm{d} m_{\mathcal{B}} / \mathrm{d} t$. The velocity cross products in the second term on the right-hand side in Eq. (32) can be simplified as follows

$$
\begin{aligned}
\vec{v}_{c / z / \mathrm{A}} \times \vec{v}_{z / w / \mathrm{A}}+\vec{v}_{z / w / \mathrm{A}} \times \vec{v}_{c / w / \mathrm{A}} & =\vec{v}_{c / z / \mathrm{A}} \times \vec{v}_{z / w / \mathrm{A}}+\vec{v}_{w / c / \mathrm{A}} \times \vec{v}_{z / w / \mathrm{A}} \\
& =\left(\vec{v}_{c / z / \mathrm{A}}+\vec{v}_{w / c / \mathrm{A}}\right) \times \vec{v}_{z / w / \mathrm{A}} \\
& =\left(\vec{v}_{c / w / \mathrm{A}}+\vec{v}_{w / z / \mathrm{A}}+\vec{v}_{w / c / \mathrm{A}}\right) \times \vec{v}_{z / w / \mathrm{A}} \\
& =-\vec{v}_{z / w / \mathrm{A}} \times \vec{v}_{z / w / \mathrm{A}}=0 .
\end{aligned}
$$

Therefore, Eq. (32) becomes

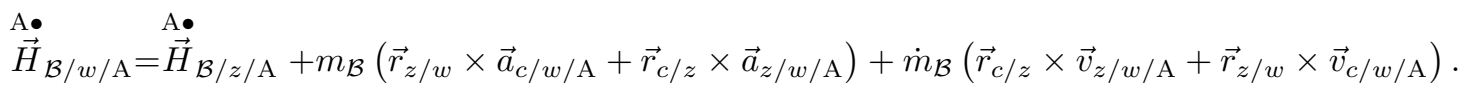

Substituting Eq. (34) into Eq. (30) yields

$$
\vec{M}_{\mathcal{B} / z}=\stackrel{\mathrm{A} \cdot}{\vec{H}_{\mathcal{B} / z / \mathrm{A}}}+m_{\mathcal{B}} \vec{r}_{c / z} \times \vec{a}_{z / w / \mathrm{A}}+\dot{m}_{\mathcal{B}} \vec{r}_{c / z} \times \vec{v}_{z / w / \mathrm{A}}+\vec{r}_{z / w} \times\left(m_{\mathcal{B}} \vec{a}_{c / w / \mathrm{A}}+\dot{m}_{\mathcal{B}} \vec{v}_{c / w / \mathrm{A}}-\vec{f}_{\mathcal{B}}\right) .
$$

Since $c$ is the center of mass of the body $\mathcal{B}$, the translational momentum of $\mathcal{B}$ relative to $w$ with respect to

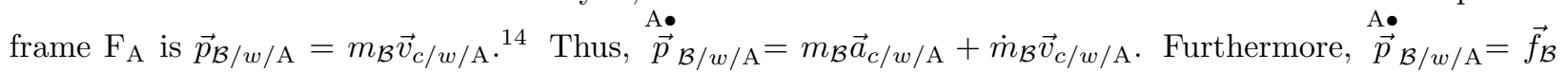
since $\mathrm{F}_{\mathrm{A}}$ is an inertial frame and $w$ is an unforced particle. Consequently, the last term on the right-hand side in Eq. (35) is zero and Eq. (35) becomes

$$
\vec{M}_{\mathcal{B} / z}=\stackrel{\mathrm{A} \cdot}{=} \vec{H}_{\mathcal{B} / z / \mathrm{A}}+\vec{r}_{c / z} \times\left(m_{\mathcal{B}} \vec{a}_{z / w / \mathrm{A}}+\dot{m}_{\mathcal{B}} \vec{v}_{z / w / \mathrm{A}}\right) .
$$

Using the transport theorem and introducing the body-fixed frame $\mathrm{F}_{\mathrm{B}}$ with mutually perpendicular frame vectors $\left(\hat{b}_{1}, \hat{b}_{2}, \hat{b}_{3}\right)$, Eq. (36) becomes

$$
\begin{aligned}
\vec{M}_{\mathcal{B} / z}= & \stackrel{\vec{H}_{\mathcal{B} / z / \mathrm{A}}}{ }+\vec{\omega}_{\mathrm{B} / \mathrm{A}} \times \vec{H}_{\mathcal{B} / z / \mathrm{A}}+\vec{r}_{c / z} \times\left[m _ { \mathcal { B } } \left(\vec{a}_{z / c / \mathrm{B}}+2 \vec{\omega}_{\mathrm{B} / \mathrm{A}} \times \vec{v}_{z / c / \mathrm{B}}+\vec{\alpha}_{\mathrm{B} / \mathrm{A}} \times \vec{r}_{z / c}\right.\right. \\
& \left.\left.+\vec{\omega}_{\mathrm{B} / \mathrm{A}} \times\left(\vec{\omega}_{\mathrm{B} / \mathrm{A}} \times \vec{r}_{z / c}\right)+\vec{a}_{c / w / \mathrm{B}}\right)+\dot{m}_{\mathcal{B}}\left(\vec{v}_{z / c / \mathrm{B}}+\vec{\omega}_{\mathrm{B} / \mathrm{A}} \times \vec{r}_{z / c}+\vec{v}_{c / w / \mathrm{A}}\right)\right],
\end{aligned}
$$

where $\vec{\omega}_{\mathrm{B} / \mathrm{A}}$ and $\vec{\alpha}_{\mathrm{B} / \mathrm{A}}:=\stackrel{\mathrm{A}}{\boldsymbol{\omega}}_{\mathrm{B} / \mathrm{A}}=\stackrel{\mathrm{B}}{\boldsymbol{\omega}}_{\mathrm{B} / \mathrm{A}}$ are the physical angular velocity and angular acceleration vectors, respectively, of frame $\mathrm{F}_{\mathrm{B}}$ relative to frame $\mathrm{F}_{\mathrm{A}}$. Again using the fact that $m_{\mathcal{B}} \vec{a}_{c / w / \mathrm{A}}+\dot{m}_{\mathcal{B}} \vec{v}_{c / w / \mathrm{A}}=\vec{f}_{\mathcal{B}}$, Eq. (37) may be rewritten as follows

$$
\begin{aligned}
\vec{M}_{\mathcal{B} / z}= & \stackrel{\mathrm{B}}{\vec{H}}_{\mathcal{B} / z / \mathrm{A}}+\vec{\omega}_{\mathrm{B} / \mathrm{A}} \times \vec{H}_{\mathcal{B} / z / \mathrm{A}}+\vec{r}_{c / z} \times\left[m _ { \mathcal { B } } \left(\vec{a}_{z / c / \mathrm{B}}+2 \vec{\omega}_{\mathrm{B} / \mathrm{A}} \times \vec{v}_{z / c / \mathrm{B}}+\vec{\alpha}_{\mathrm{B} / \mathrm{A}} \times \vec{r}_{z / c}\right.\right. \\
& \left.\left.+\vec{\omega}_{\mathrm{B} / \mathrm{A}} \times\left(\vec{\omega}_{\mathrm{B} / \mathrm{A}} \times \vec{r}_{z / c}\right)\right)+\dot{m}_{\mathcal{B}}\left(\vec{v}_{z / c / \mathrm{B}}+\vec{\omega}_{\mathrm{B} / \mathrm{A}} \times \vec{r}_{z / c}\right)+\vec{f}_{\mathcal{B}}\right] .
\end{aligned}
$$

$\vec{H}_{\mathcal{B} / z / \mathrm{A}}$ may be expressed using the physical inertia matrix $\vec{I}_{\mathcal{B} / z}$ of the body $\mathcal{B}$ relative to the point $z$ : $\vec{H}_{\mathcal{B} / z / \mathrm{A}}=\vec{I}_{\mathcal{B} / z} \vec{\omega}_{\mathrm{B} / \mathrm{A}}$. Therefore, Eq. (38) becomes

$$
\begin{aligned}
& \vec{M}_{\mathcal{B} / z}=\stackrel{\mathrm{B}}{\vec{I}}_{\mathcal{B} / z} \vec{\omega}_{\mathrm{B} / \mathrm{A}}+\vec{I}_{\mathcal{B} / z} \vec{\alpha}_{\mathrm{B} / \mathrm{A}}+\vec{\omega}_{\mathrm{B} / \mathrm{A}} \times\left(\vec{I}_{\mathcal{B} / z} \vec{\omega}_{\mathrm{B} / \mathrm{A}}\right)-\vec{r}_{c / z} \times\left[m _ { \mathcal { B } } \left(\vec{a}_{c / z / \mathrm{B}}+2 \vec{\omega}_{\mathrm{B} / \mathrm{A}} \times \vec{v}_{c / z / \mathrm{B}}\right.\right. \\
& \left.\left.+\vec{\alpha}_{\mathrm{B} / \mathrm{A}} \times \vec{r}_{c / z}+\vec{\omega}_{\mathrm{B} / \mathrm{A}} \times\left(\vec{\omega}_{\mathrm{B} / \mathrm{A}} \times \vec{r}_{c / z}\right)\right)+\dot{m}_{\mathcal{B}}\left(\vec{v}_{c / z / \mathrm{B}}+\vec{\omega}_{\mathrm{B} / \mathrm{A}} \times \vec{r}_{c / z}\right)-\vec{f}_{\mathcal{B}}\right] .
\end{aligned}
$$

This equation describes the general angular motion of a rigid body $\mathcal{B}$ with center of mass $c$ and timevarying mass and inertia properties. Eq. (39) is now resolved in the body-fixed frame $\mathrm{F}_{\mathrm{B}}$ for the example 
axisymmetric spacecraft in Fig. 1. $\mathrm{F}_{\mathrm{B}}$ is assumed to be the principal frame as well. The inertia matrix of the axisymmetric spacecraft $\mathcal{B}$ relative to the body-fixed point $z$ is expressed in frame $\mathrm{F}_{\mathrm{B}}$ as

$$
J_{z}:=\left.\vec{I}_{\mathcal{B} / z}\right|_{\mathrm{B}}=\left[\begin{array}{ccc}
J_{\mathrm{T}} & 0 & 0 \\
0 & J_{\mathrm{T}} & 0 \\
0 & 0 & J_{\mathrm{R}}
\end{array}\right]
$$

The angular velocity vector is resolved in $\mathrm{F}_{\mathrm{B}}$ as $\left.\vec{\omega}_{\mathrm{B} / \mathrm{A}}\right|_{\mathrm{B}}=\left[\omega_{1}, \omega_{2}, \omega_{3}\right]^{\mathrm{T}}$, where $\omega_{1}, \omega_{2}$, and $\omega_{3}$ are the angular velocity vector projections on the principal axes of $\mathcal{B}$. Likewise, $\left.\vec{\alpha}_{\mathrm{B} / \mathrm{A}}\right|_{\mathrm{B}}=\left[\dot{\omega}_{1}, \dot{\omega}_{2}, \dot{\omega}_{3}\right]^{\mathrm{T}},\left.\vec{M}_{\mathcal{B} / z}\right|_{\mathrm{B}}=$ $\left[M_{1}, M_{2}, M_{3}\right]^{\mathrm{T}},\left.\vec{f}_{\mathcal{B}}\right|_{\mathrm{B}}=\left[f_{1}, f_{2}, f_{3}\right]^{\mathrm{T}},\left.\vec{a}_{c / z / \mathrm{B}}\right|_{\mathrm{B}}=\left[a_{1}, a_{2}, a_{3}\right]^{\mathrm{T}},\left.\vec{v}_{c / z / \mathrm{B}}\right|_{\mathrm{B}}=\left[v_{1}, v_{2}, v_{3}\right]^{\mathrm{T}}$, and $\left.\vec{r}_{c / z}\right|_{\mathrm{B}}=\left[r_{1}, r_{2}, r_{3}\right]^{\mathrm{T}}$. We assume that the spacecraft's mass changes with a constant rate

$$
m_{\mathcal{B}}(t)=m_{\mathcal{B}, 0}-\left(\dot{m}_{\mathrm{ox}}+\dot{m}_{\mathrm{f}}\right) t,
$$

where $m_{\mathcal{B}, 0}, \dot{m}_{\mathrm{ox}}$, and $\dot{m}_{\mathrm{f}}$ are constant scalars. Note that $\dot{m}_{\mathrm{ox}}$ and $\dot{m}_{\mathrm{f}}$ are the mass flow rates of the oxidizer and fuel, respectively. In analogy to Eq. (41), the time-varying masses of the fuel and oxidizer are

$$
\begin{gathered}
m_{\mathrm{f}}(t)=m_{\mathrm{f}, 0}-\dot{m}_{\mathrm{f}} t, \\
m_{\mathrm{ox}}(t)=m_{\mathrm{ox}, 0}-\dot{m}_{\mathrm{ox}} t,
\end{gathered}
$$

where $m_{\mathrm{f}, 0}$ and $m_{\mathrm{ox}, 0}$ are the initial fuel and oxidizer masses. The time-varying lengths of the remaining fuel $l_{\mathrm{f}}$ and oxidizer $l_{\mathrm{ox}}$ (see Fig. 1) are

$$
\begin{gathered}
l_{\mathrm{f}}(t)=l_{\mathrm{f}, 0}-i_{\mathrm{f}} t, \\
l_{\mathrm{ox}}(t)=l_{\mathrm{ox}, 0}-i_{\mathrm{ox}} t,
\end{gathered}
$$

where $\dot{l}_{\mathrm{f}}=\dot{m}_{\mathrm{f}} /\left(\pi r_{\mathrm{e}}^{2} \rho_{\mathrm{f}}\right)$ and $\dot{l}_{\mathrm{ox}}=\dot{m}_{\mathrm{ox}} /\left(\pi r_{\mathrm{e}}^{2} \rho_{\mathrm{ox}}\right)$ are constant scalars. Here, $\rho_{\mathrm{f}}$ and $\rho_{\mathrm{ox}}$ are the fuel's and oxidizer's density, respectively. The parameter $r_{\mathrm{e}}$ is the radius of the engine and tank section of the spacecraft as shown in Fig. 1. It is assumed that the center of mass is always on the symmetry axis $\left(\hat{b}_{3}\right.$-axis as shown in Fig. 1). Therefore, the first and second component of $\left.\vec{r}_{z / c}\right|_{\mathrm{B}},\left.\vec{v}_{z / c / \mathrm{B}}\right|_{\mathrm{B}}$, and $\left.\vec{a}_{z / c / \mathrm{B}}\right|_{\mathrm{B}}$ are zero: $r_{1}=r_{2}=0$, $v_{1}=v_{2}=0$, and $a_{1}=a_{2}=0$. Using Eq. (41) to Eq. (45), the distance between the center of mass $c$ and point $z$ is given by

$$
r_{3}(t)=\frac{C_{r_{3}}+m_{\mathrm{ox}}(t)\left(l_{\mathrm{e}}+\frac{l_{\mathrm{ox}}(t)}{2}\right)+m_{\mathrm{f}}(t)\left(l_{\mathrm{e}}+l_{\mathrm{ox}, 0}+\frac{l_{\mathrm{f}}(t)}{2}\right)}{m_{\mathcal{B}}(t)},
$$

where $C_{r_{3}}=m_{\mathrm{e}} l_{\mathrm{e}} / 2+m_{\mathrm{p}}\left(l_{\mathrm{e}}+l_{\mathrm{ox}, 0}+l_{\mathrm{f}, 0}+l_{\mathrm{p}} / 2\right)$ is a constant scalar. $m_{\mathrm{e}}$ and $m_{\mathrm{p}}$ denote the masses of the engine and the payload, respectively. The velocity of the center of mass $c$ relative to point $z$ with respect to frame $\mathrm{F}_{\mathrm{B}}$ is given by $v_{3}=\dot{r}_{3}$ reading

$$
v_{3}(t)=\frac{C_{v_{3}}-m_{\mathrm{ox}}(t) \frac{i_{\mathrm{ox}}}{2}-\dot{m}_{\mathrm{ox}} \frac{l_{\mathrm{ox}}(t)}{2}-m_{\mathrm{f}}(t) \frac{i_{\mathrm{f}}}{2}-\dot{m}_{\mathrm{f}} \frac{l_{\mathrm{f}}(t)}{2}+r_{3}(t)\left(\dot{m}_{\mathrm{ox}}+\dot{m}_{\mathrm{f}}\right)}{m_{\mathcal{B}}(t)},
$$

where $C_{v_{3}}=-\dot{m}_{\mathrm{ox}} l_{\mathrm{e}}-\dot{m}_{\mathrm{f}}\left(l_{\mathrm{e}}+l_{\mathrm{ox}, 0}\right)$ is a constant scalar. Likewise, the acceleration of the center of mass $c$ relative to point $z$ with respect to frame $\mathrm{F}_{\mathrm{B}}$ is given by $a_{3}=\dot{v}_{3}=\ddot{r}_{3}$ which yields

$$
a_{3}(t)=\frac{C_{a_{3}}+2 v_{3}(t)\left(\dot{m}_{\mathrm{ox}}+\dot{m}_{\mathrm{f}}\right)}{m_{\mathcal{B}}(t)}
$$

where $C_{a_{3}}=\dot{m}_{\mathrm{ox}} \dot{l}_{\mathrm{ox}}+\dot{m}_{\mathrm{f}} \dot{l}_{\mathrm{f}}$ is a constant scalar. The time-dependent components of the inertia matrix in Eq. (40) follow from the parallel axis theorem. The principal moment of inertia about the $\hat{b}_{3}$-axis relative to point $z$ is given by

$$
J_{\mathrm{R}}(t)=\frac{1}{2}\left(C_{J_{\mathrm{R}}}+r_{\mathrm{e}}^{2}\left[m_{\mathrm{ox}}(t)+m_{\mathrm{f}}(t)\right]\right),
$$

where $C_{J_{\mathrm{R}}}=r_{\mathrm{e}}^{2} m_{\mathrm{e}}+r_{\mathrm{p}}^{2} m_{\mathrm{p}}$ is a constant scalar. The parameter $r_{\mathrm{p}}$ is the radius of the payload section. The principal moment of inertia about the $\hat{b}_{1}$ - and $\hat{b}_{2}$ axis is

$$
J_{\mathrm{T}}(t)=C_{J_{\mathrm{T}}}+m_{\mathrm{ox}}(t)\left(\frac{r^{2}}{4}+\frac{l_{\mathrm{ox}}^{2}(t)}{12}+\left[l_{\mathrm{e}}+\frac{l_{\mathrm{ox}}(t)}{2}\right]^{2}\right)+m_{\mathrm{f}}(t)\left(\frac{r^{2}}{4}+\frac{l_{\mathrm{f}}^{2}(t)}{12}+\left[l_{\mathrm{e}}+l_{\mathrm{ox}, 0}+\frac{l_{\mathrm{f}}(t)}{2}\right]^{2}\right),
$$


where $C_{J_{\mathrm{T}}}=m_{\mathrm{e}}\left(\frac{r_{\mathrm{e}}^{2}}{4}+\frac{l_{\mathrm{e}}^{2}}{3}\right)+m_{\mathrm{p}}\left(\frac{r_{\mathrm{p}}^{2}}{4}+\frac{l_{\mathrm{p}}^{2}}{12}+\left[l_{\mathrm{e}}+l_{\mathrm{ox}, 0}+l_{\mathrm{f}, 0}+\frac{l_{\mathrm{p}}}{2}\right]^{2}\right)$ is a constant scalar. The time derivatives with respect to frame $\mathrm{F}_{\mathrm{B}}$ of the principal moment of inertia are given by

$$
\begin{gathered}
\dot{J}_{\mathrm{R}}=-\frac{r_{\mathrm{e}}^{2}}{2}\left(\dot{m}_{\mathrm{ox}}+\dot{m}_{\mathrm{f}}\right), \\
\dot{J}_{\mathrm{T}}(t)=C_{j_{\mathrm{T}}}-\left(l_{\mathrm{e}}+\frac{l_{\mathrm{ox}}(t)}{3}\right)\left(\dot{m}_{\mathrm{ox}} l_{\mathrm{ox}}(t)+m_{\mathrm{ox}}(t) \dot{l}_{\mathrm{ox}}\right)-\left(l_{\mathrm{e}}+l_{\mathrm{ox}, 0}+\frac{l_{\mathrm{f}}(t)}{3}\right)\left(\dot{m}_{\mathrm{ox}} l_{\mathrm{f}}(t)+m_{\mathrm{f}}(t) \dot{l}_{\mathrm{f}}\right) \\
-\frac{m_{\mathrm{ox}}(t) l_{\mathrm{ox}}(t) \dot{l}_{\mathrm{ox}}+m_{\mathrm{f}}(t) l_{\mathrm{f}}(t) \dot{l}_{\mathrm{f}}}{3}
\end{gathered}
$$

where $C_{\dot{J}_{\mathrm{T}}}=-\dot{m}_{\mathrm{ox}}\left(r_{\mathrm{e}}^{2} / 4+l_{\mathrm{e}}^{2}\right)-\dot{m}_{\mathrm{f}}\left(r_{\mathrm{e}}^{2} / 4+\left(l_{\mathrm{e}}+l_{\mathrm{ox}, 0}\right)^{2}\right)$ is a constant scalar. Note that $\dot{J}_{\mathrm{R}}$ in Eq. (51) is a constant scalar. The equations of motion for the example axisymmetric spacecraft with time-varying mass/inertia properties follow from Eq. (39), yielding

$$
\begin{gathered}
\dot{\omega}_{1}=\frac{M_{1}+\left(2 r_{3}(t) v_{3}(t) m_{\mathcal{B}}(t)+r_{3}^{2}(t) \dot{m}_{\mathcal{B}}-\dot{J}_{\mathrm{T}}(t)\right) \omega_{1}+\left(J_{\mathrm{T}}(t)-J_{\mathrm{R}}(t)-r_{3}^{2}(t) m_{\mathcal{B}}(t)\right) \omega_{2} \omega_{3}-r_{3}(t) f_{2}}{J_{\mathrm{T}}(t)-r_{3}^{2}(t) m_{\mathcal{B}}(t)}, \\
\dot{\omega}_{2}=\frac{M_{2}+\left(2 r_{3}(t) v_{3}(t) m_{\mathcal{B}}(t)+r_{3}^{2}(t) \dot{m}_{\mathcal{B}}-\dot{J}_{\mathrm{T}}(t)\right) \omega_{2}+\left(J_{\mathrm{R}}(t)-J_{\mathrm{T}}(t)+r_{3}^{2}(t) m_{\mathcal{B}}(t)\right) \omega_{1} \omega_{3}+r_{3}(t) f_{1}}{J_{\mathrm{T}}(t)-r_{3}^{2}(t) m_{\mathcal{B}}(t)}, \\
\dot{\omega}_{3}=\frac{M_{3}-\omega_{3} \dot{J}_{\mathrm{R}}}{J_{\mathrm{R}}(t)},
\end{gathered}
$$

where $\dot{m}_{\mathcal{B}}=\dot{m}_{\text {ox }}+\dot{m}_{\mathrm{f}}$ is a constant scalar. Note that we explicitly state the time dependence of $m_{\mathcal{B}}, r_{3}, v_{3}$, $J_{\mathrm{R}}, J_{\mathrm{T}}$, and $\dot{J}_{\mathrm{T}}$ in Eq. (53) to Eq. (55). It is self-evident that the state variables $\omega_{1}, \omega_{2}$, and $\omega_{3}$ as well as $\dot{\omega}_{1}, \dot{\omega}_{2}$, and $\dot{\omega}_{3}$ are time-dependent too. The same may be true for the components of the moment $M_{1}, M_{2}$, and $M_{3}$ as well as for the components of the total force $f_{1}, f_{2}$, and $f_{3}$.

In the following we assume that $M_{3}=0$. Then the solution for $\omega_{3}$ is readily obtained since Eq. (55) is decoupled from Eq. (53) and (54)

$$
\omega_{3}(t)=\frac{\omega_{3,0} J_{\mathrm{R}, 0}}{J_{\mathrm{R}, 0}+\dot{J}_{\mathrm{R}} t}
$$

where $\dot{J}_{\mathrm{R}}$ is given by Eq. (51), $J_{\mathrm{R}, 0}=J_{\mathrm{R}}(t=0)=\left(r_{\mathrm{e}}^{2}\left(m_{\mathrm{ox}, 0}+m_{\mathrm{f}, 0}\right)+C_{J_{\mathrm{R}}}\right) / 2$ is the initial principal angular momentum about the symmetry axis, and $\omega_{3,0}$ is the initial angular velocity about the symmetry axis. The number of differential equations describing the system may be reduced by substituting Eq. (56) into Eq. (53) and (54).

\section{Acknowledgments}

This research is supported by the National Science Foundation Award Number EECS 1404814.

\section{References}

\footnotetext{
${ }^{1}$ Kolmanovsky, I. V. and Maizenberg, T. L., "Optimal Containment Control for a Class of Stochastic Systems Perturbed by Poisson and Wiener Processes," IEEE Transactions on Automatic Control, Vol. 47, No. 12, 2002, pp. 2041-2046.

${ }^{2}$ Kolmanovsky, I. V., Lezhnev, L., and Maizenberg, T. L., "Discrete-Time Drift Counteraction Stochastic Optimal Control: Theory and Application-Motivated Examples," Automatica, Vol. 44, No. 1, 2008, pp. 177-184.

${ }^{3}$ Kolmanovsky, I. V. and Menezes, A. A., "A Stochastic Drift Counteraction Optimal Control Approach to Glider Flight Management," Proceedings of the 2011 American Control Conference (ACC), San Francisco, CA, June 2011.

${ }^{4}$ Zidek, R. A. E. and Kolmanovsky, I. V., "Deterministic Drift Counteraction Optimal Control and Its Application to Satellite Life Extension," Proceedings of the 54th IEEE Conference on Decision and Control, Osaka, Japan, December 2015.

${ }^{5}$ Bertsekas, D. P., Dynamic Programming and Optimal Control, Vol. I, Athena Scientific, Belmont, MA, 2005.

${ }^{6}$ Bertsekas, D. P., "Infinite-time Reachability of State-Space Regions by Using Feedback Control," IEEE Transactions on Automatic Control, Vol. 17, No. 5, 1972, pp. 604-613.

${ }^{7}$ Blanchini, F. and Miani, S., Set-Theoretic Methods in Control, Birkhäuser, Boston, MA, 2008.

${ }^{8}$ Powell, W. B., Approximate Dynamic Programming, Solving the Curses of Dimensionality, John Wiley and Sons, Hoboken, NJ, 2011.
} 
${ }^{9}$ Bertsekas, D. P., Dynamic Programming and Optimal Control, Vol. II: Approximate Dynamic Programming, Athena Scientific, Belmont, MA, 2012.

${ }^{10}$ Noll, R. B., Zvara, J., and Deyst, J. J., "Spacecraft Attitude Control During Thrusting Maneuvers," Tech. Rep. NASA SP-8059, National Aeronautics and Space Administration, Washington, D.C., 1971.

${ }^{11}$ Tsiotras, P. and Longuski, J. M., "A New Parameterization of the Attitude Kinematics," Journal of the Astronautical Sciences, Vol. 43, No. 3, 2008, pp. 243-262.

${ }^{12}$ Huzel, D. K. and Huang, D. H., "Design of Liquid Propellant Rocket Engines," Tech. Rep. NASA SP-125, Rocketdyne Division, North American Aviation, Inc., Washington, D.C., 1967.

${ }^{13}$ Sutton, G. P., History of Liquid Propellant Rocket Engines, American Institute of Aeronautics and Astronautics, Inc., Reston, VA, 2006.

${ }^{14}$ Bernstein, D. S., Geometry, Kinematics, Statics, and Dynamcis, Princeton University Press, Princeton and Oxford, 2012 .

18 of 18 